\title{
Thermally-Induced Fracture Analysis of Polycrystalline Materials by Using Peridynamics
}

\author{
Mingyang $\mathrm{Li}^{\mathrm{a}}$, Wei Lu ${ }^{\mathrm{a}, \mathrm{b}}$, Erkan Oterkus ${ }^{\mathrm{a}, *}$ and Selda Oterkus ${ }^{\mathrm{a}}$ \\ aPeriDynamics Research Centre, Department of Naval Architecture, Ocean and Marine \\ Engineering, University of Strathclyde, Glasgow, United Kingdom \\ ${ }^{\mathrm{b}}$ College of Shipbuilding Engineering, Harbin Engineering University, Harbin, China
}

\begin{abstract}
Polycrystalline materials are widely utilized in engineering fields. In this study, peridynamic (PD) models are developed for the first time in the literature to investigate thermally-induced fracture phenomenon for cubic polycrystals and ceramic made of different materials. After validating the current model, the influences of the grain size, grain boundary strength and composition of the materials on the fracture behavior are analyzed. Two different types of pre-existing cracks, i.e. vertical and horizontal, are considered. The effect of grain size is much more obvious for ceramic materials and crack branching is observed for both vertical and horizontal crack cases. Grain boundary strength has a significant influence on crack behaviour. For weaker grain boundaries intergranular fracture pattern is observed whereas for stronger grain boundaries transgranular crack pattern is more dominant. Crack branching is much more significant when the silicon carbide ratio is higher due to the difference in coefficients of thermal expansion of two different materials. By comparing with the reference results available in the literature similar fracture features are obtained.
\end{abstract}

Keywords: Fracture; peridynamics; cubic polycrystal; thermal loading; ceramic

\section{Introduction}

Most metals, alloys and ceramics are usually polycrystalline materials. Polycrystalline materials usually consist of numerous crystals at the microscale. Each crystal may have its own properties such as shape, size, grain orientation and so forth. These microscale properties can have an impact on the features of materials at the macroscale and finally affect the characteristics of the whole structure. Although research studies concerning the polycrystalline materials have been performed for decades, understanding the fracture phenomenon in these materials, especially at the microscale, is rather limited. This is because not only the crystals have various characteristics, but also the patterns of the crack can also be diverse [1].

Experiments are powerful tools to carry out research about polycrystalline materials. Traditional experiments, as a result of lacking advanced photographic and imaging technologies, usually concentrated on one specific aspect of polycrystals [2-4]. Recently, with the development of tomography, even the 3-D microstructures of the polycrystalline materials can be clearly observed. By utilizing a dual-beam focused iron beam-scanning electron microscope (FIB-SEM) combining with an Electron Backscattered Diffraction (EBSD) system, Groeber et al. [5] successfully obtained the information about grain size and orientations of the nickel-based superalloy in 3-D. By accurately collecting data about grains, the mechanical performance of the material can be estimated and tested. Ludwig et al. [6] used the x-ray diffraction contrast tomography (DCT) to obtain the shapes and orientations of the grains of a

\footnotetext{
* Corresponding author at: PeriDynamics Reserch centre, Department of Naval Architecture, Ocean and Marine Engineering, University of Strathclyde, 100 Montrose Street, Glasgow, G4 OLZ, UK

Email addresses: m.li@strath.ac.uk (M. Li),wei.lu@strath.ac.uk (W. Lu), erkan.oterkus@strath.ac.uk

(E. Oterkus), selda.oterkus@strath.ac.uk (S. Oterkus)
} 
polycrystalline material without considering deformations. Then, by using the same tomography technique together with propagation-based phase-contrast tomography, Herbig et al. [7] investigated the microstructure of the titanium alloy. More importantly, this setup also showed the potential to observe crack propagation. Although experiments can provide important information about the microstructure of polycrystalline materials, experiments about the fracture of polycrystals usually require expensive equipment and the preparation and analysis process are quite complex and time-consuming [8]. Computational modeling of polycrystalline materials would be a more practical and economical approach.

Cohesive zone model $(\mathrm{CZM})$ is one of the most popular numerical methods to simulate the fracture of polycrystalline materials. After applying the Finite Element Method (FEM) to CZM, Camacho and Ortiz [9] simulated the dynamic fracture of brittle materials based on their linear cohesive law. Ortiz and Pandolfi [10] focused on the problem of fracture of metals and the initiation and propagation of the crack were analyzed according to the potential-based law developed by Tvergaard and Hutchinson [11]. CZM combining with FEM was also chosen by Lin et al. [12] to study about the role of the properties of the cohesive zone on the transitions of the crack pattern. Boundary element method (BEM) [13] can be a more effective and easier method than CZM and it can overcome some drawbacks of FEM including the complexity of the analysis. BEM has been successfully applied to analyze different types of fracture of polycrystalline materials $[14,15]$. Furthermore, BEM was further extended to model polycrystals to illustrate the crack propagation phenomenon under thermal loading $[8,16,17]$. Multiscale cohesive zone model (MCZM) was recently developed from CZM by Zeng and Li [18] and it is another useful and improved tool for the simulation of fracture of polycrystalline materials. Intergranular/transgranular and brittle/ductile fracture were analyzed using MCZM by Qian and Li [19] and Li et al. [20], respectively. Galvis and Sollero [21] combined the MCZM with BEM together and the analysis of the intergranular crack propagation in zinc and nickel was performed. Apart from CZM, the Extended Finite Element Method (X-FEM) and Body Force method can also be utilized to simulate the fracture process in polycrystalline materials $[22,23]$.

As an alternative approach, peridynamics (PD) can be utilized. PD was introduced by Silling [24] and its governing equations are in the form of integro-differential equations. The PD formulation does not contain any spatial derivatives which leads to advantages for problems including discontinuities such as cracks. PD has been applied to many different types of material systems including metals [25-27] and composites [28-30]. A recent review on peridynamics research can be found in Javili et al. [31].

In order to apply PD methodology to model polycrystalline materials especially to simulate fracture at microscale, several research studies have been performed for different types of crystals. First of all, Ghajari et al. [32] developed a novel model for hexagonal crystals based on Bond-based PD theory (BBPD). This model allows the phenomena of crack nucleation and branching to be automatically simulated during the numerical analysis. The model was verified by comparing PD results against analytical and experimental reference solutions. Then the fracture of the microstructure of alumina was investigated indicating that this PD model can be successfully used to analyze materials with hexagonal crystal structures. Secondly, Askari et al. [33] built a PD model for crystals with cubic systems. The effect of the grain boundary on the mechanisms of the fracture was explored for silicon using this model. De Meo et al. [1] introduced another novel model based on BBPD for cubic crystals. The 
robustness of this model was proven by both static/dynamic analysis of an iron plate without a crack and a steel plate with a pre-existing crack. The influences of the grain size, grain orientation, grain boundary strength, and fracture toughness $\left(K_{I c}\right)$ of the materials on the fracture mechanisms of steel were also explored. De Meo et al. [34] used their model to investigate the stress corrosion cracking (SCC) phenomenon contributed by adsorptioninduced decohesion. Their results were quite identical to the reference experimental results. Zhu et al. [35] extended this BBPD model to ordinary state-based PD (SBPD) which eliminates the restriction of the limitations on material constants due to assumptions of BBPD formulation.

As a well-known phenomena, structures made from metals or ceramics usually suffer from temperature change. These changes can be due to the heating-up process during the working condition or the cooling-down process during the fabrication procedure. The thermal expansion/shrinkage of the material can result in initiation and propagation of cracks especially under the condition that there is a pre-existing crack. The thermal-expansion induced fracture of polycrystalline materials are mainly caused by: 1) the differences of the thermal expansion coefficients $(\alpha)$ among different material compositions, 2) transformations of the crystal systems during the change of the temperature, 3 ) elastic anisotropy leading to variation of strain distributions [8]. This study focuses on peridynamic modelling of the fracture behaviour at the microscale for the polycrystalline materials caused by thermal loading for the first time in the literature. In Section 2, the PD model will be briefly presented. Next, three static examples will be shown as part of Section 3 to test the feasibility of the model. In Sections 3.4 and 3.5, the numerical analysis of the crack propagation for a cubic polycrystalline material and a ceramic made of two different materials will be presented. Finally, conclusions of the study will be given.

\section{PD theory}

\subsection{Basic equations}

The governing equations of PD are in the form of integro-differential equations and can be written as:

$$
\rho(\mathbf{x}) \ddot{\mathbf{u}}(\mathbf{x}, t)=\int_{H_{\mathbf{x}}} \mathbf{f}\left(\mathbf{u}\left(\mathbf{x}^{\prime}, t\right)-\mathbf{u}(\mathbf{x}, t), \mathbf{x}^{\prime}-\mathbf{x}\right) d V_{\mathbf{x}^{\prime}}+\mathbf{b}(\mathbf{x}, t)
$$

where $\rho(\mathbf{x})$ is the density of the material point, $\ddot{\mathbf{u}}(\mathbf{x}, t)$ is the acceleration of the material point at time $t ; H_{\mathbf{x}}$ is defined as the horizon (shown in Fig. 1) which defines the range of interaction of the material point at $\mathbf{x}$ with other material points; $d V_{\mathbf{x}^{\prime}}$ is the volume of the materials points within the horizon; $\mathbf{b}(\mathbf{x}, t)$ is the body load externally exerted on the material point at $\mathbf{x}$. Analytical solution of Eq. (1a) is usually not available. Therefore, meshless approach is widely utilized to numerically solve Eq. (1a) which then takes the discretized form as:

$$
\rho\left(\mathbf{x}_{i}\right) \ddot{\mathbf{u}}\left(\mathbf{x}_{i}, t\right)=\sum_{j=1}^{N} \mathbf{f}\left(\mathbf{u}\left(\mathbf{x}_{j}, t\right)-\mathbf{u}\left(\mathbf{x}_{i}, t\right), \mathbf{x}_{j}-\mathbf{x}_{i}\right) V_{j}+\mathbf{b}\left(\mathbf{x}_{i}, t\right)
$$


where $N$ is the number of material points withing the horizon of the material point $\mathbf{x}_{i} . \mathbf{f}$ is the bond (interaction) force between material points $\mathbf{x}_{i}$ and $\mathbf{x}_{j}$ and it can be calculated as:

$$
\mathbf{f}=C S \frac{\mathbf{y}_{j}-\mathbf{y}_{i}}{\left|\mathbf{y}_{j}-\mathbf{y}_{i}\right|}
$$

where $C$ is the bond constant between two material points. For 2-D isotropic materials, the bond constant can be written as [36]:

$$
c=\frac{12 k}{\pi h \delta^{3}}
$$

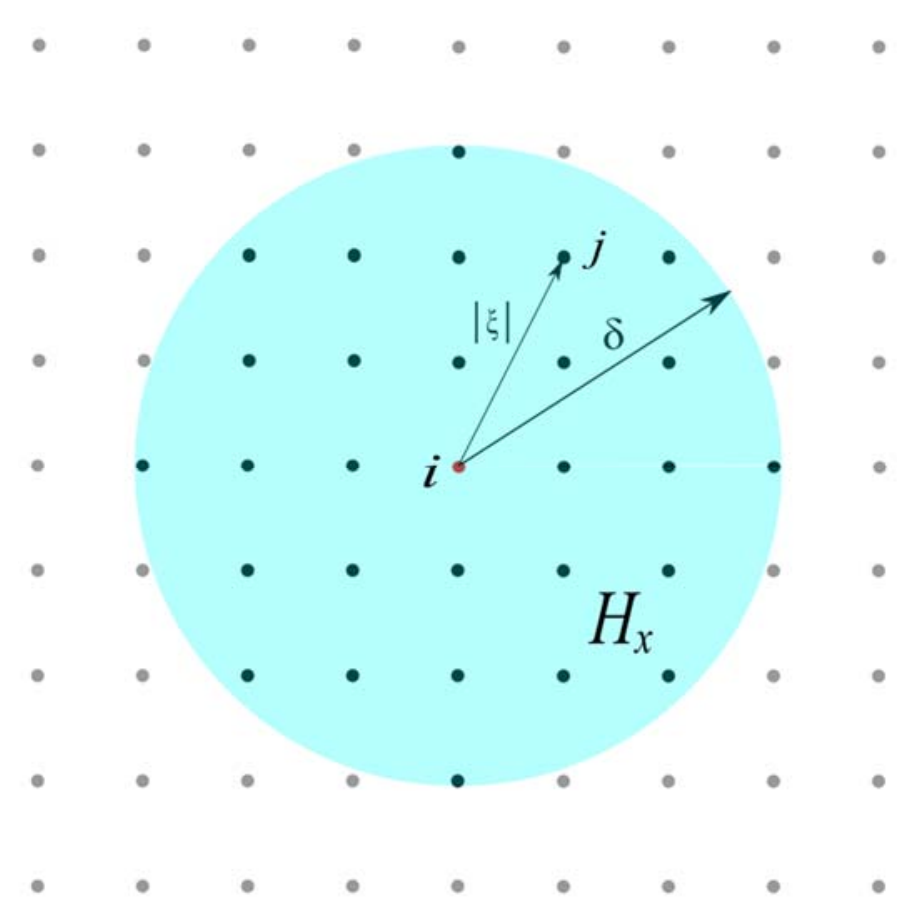

Fig. 1. PD horizon and interactions between material points

In Eq. (3), $k$ is the bulk modulus, i.e. $k=E / 2(1-v)$, and $h$ is the thickness of the plate. $\delta$ is the radius of the horizon, usually taken as $\delta=3 \times d x$ where $\mathrm{dx}$ is the grid size [36]. $E$ and $v$ are material constants corresponding to elastic modulus and Poisson's ratio, respectively. It should be noted that $v$ is constrained as $1 / 3$ for 2-D problems due to the limitation of BBPD. $\mathbf{y}=\mathbf{x}+\mathbf{u}$ is the position of the material point in the deformed configuration where $\mathbf{u}$ is the displacement and $\mathbf{x}$ is the position of the material point in the original configuration. If the temperature loading is taken into account, the equation of the PD force can be updated as:

$$
\mathbf{f}=(c s-\alpha \Delta T) \frac{\mathbf{y}_{j}-\mathbf{y}_{i}}{\left|\mathbf{y}_{j}-\mathbf{y}_{i}\right|}
$$

where $\Delta T$ is the temperature change of the bond. The temperature change of the bond can be calculated by averaging the temperature changes of the two interacting material points. 
One of the most important parameters of BBPD is the stretch and it can be defined as:

$$
S=\frac{\left|\mathbf{y}_{j}-\mathbf{y}_{i}\right|-\left|\mathbf{x}_{j}-\mathbf{x}_{i}\right|}{\left|\mathbf{x}_{j}-\mathbf{x}_{i}\right|}
$$

In order to judge whether a bond should be broken or not, critical stretch $\left(s_{0}\right)$ parameter is utilised. If the value of stretch is larger than critical stretch, the bond can be regarded as broken, i.e. there will not be any force acting between the two material points of this bond. The critical stretch can be calculated from the material properties as:

$$
s_{0}=\sqrt{\frac{4 \pi G_{c}}{9 E \delta}}
$$

where $G_{c}$ is the critical energy release rate.

2.2 PD equations for single cubic crystal

Under the plane-stress condition, the local stiffness matrix for a single cubic crystal can be written as:

$$
[c]=\left[\begin{array}{ccc}
c_{11} & c_{12} & 0 \\
c_{12} & c_{11} & 0 \\
0 & 0 & c_{44}
\end{array}\right]
$$

Fig. 2. PD model for polycrystalline when the orientation of the crystal is $0^{\circ}$ (blue lines represent Type- 1 bonds and red lines represent Type- 2 bonds)

Based on the PD model developed by De Meo et al. [1], bonds for a single cubic crystal can be divided into two different groups. Type-1 bonds shown as blue lines in Fig. 2 are located in all directions of the material. The bond constant for Type- 1 bonds can be calculated from the components of the stiffness matrix as: 


$$
c_{T 1}=\frac{12\left(c_{11}^{2}-c_{11} c_{12}\right)}{\pi h \delta^{3} c_{11}}
$$

Type- 2 bonds shown as red lines in Fig. 2 exist in some specific directions. These directions are defined according to the orientations of the crystals. Type- 2 bond angle with respect to the crystal orientation is specified as $\frac{\pi}{4}(2 \times i-1),(i=1,2,3,4)$. The bond constant for Type- 2 bonds can be calculated as:

$$
c_{T 2}=\frac{4\left(3 c_{11} c_{12}-2 c_{12}{ }^{2}-c_{11}^{2}\right)}{\beta c_{11}}
$$

where $\beta=\sum_{j=1}^{N} \xi_{i j} V_{j}, \xi_{i j}$ is the bond length between material points $i$ and $j$.

The accuracy of this model under mechanical loading was verified by De Meo et al. [1]. For cubic crystal, the thermal expansion coefficient, $\alpha$ is same in all directions. Therefore, the thermal loading can be directly taken into account by using the bond force expression given in Eq. (4).

\subsection{PD model for polycrystalline materials}

In order to generate the polycrystalline model, the Voronoi tessellation method was utilized. The seeds were randomly distributed over the plate and the nodes were divided into different crystals by minimizing the distance between the seeds and nodes. Besides, for cubic polycrystals, the grain size and grain orientations were randomly determined. The plot of the crystals is shown in Fig. 3. In this study, the grain boundary width is assumed to be zero. Moreover, for the bonds crossing the grain boundaries, their bond constant values are calculated as the average of the bond constant values of the associated material points. One definition that should be noted here is the grain boundary coefficient (GBC) and it is the ratio of the strength of the bond crossing the grain boundary to the strength of the bond inside the grains. It is an important parameter for the investigation of the influence of the grain boundary strength.

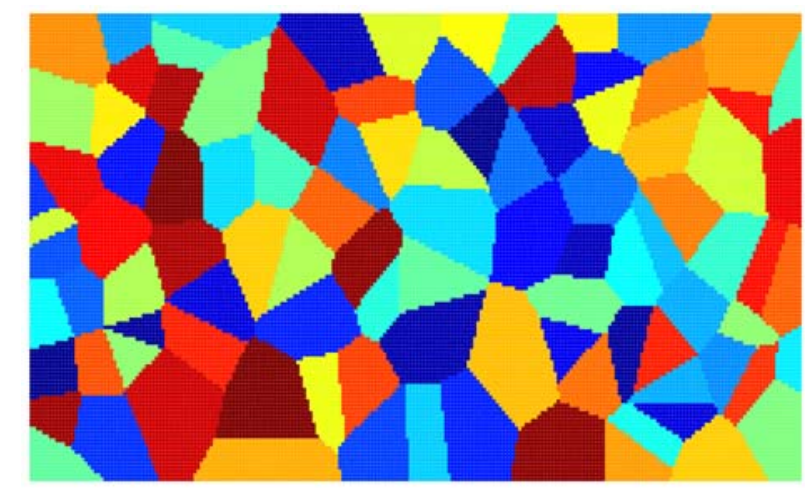

Fig. 3. Crystals generated by the Voronoi tessellation method. 


\section{Numerical analysis}

In this section, several numerical cases are considered to verify the current formulation and demonstrate its capabilities. First, static analysis of an isotropic plate subjected to thermal loading problem is considered to verify the thermo-mechanical peridynamic formulation. Next, the same analysis is repeated by considering a single cubic crystal and cubic polycrystals instead of an isotropic material. The solution to the static cases is obtained by utilising an implicit scheme by directly equating the inertia term to 0 in the peridynamic equation of motion given in Eqs. $(1 \mathrm{a}, \mathrm{b})$ and solving a matrix system to obtain the solution as explained in [37].

Following static analyses, dynamic analysis of cubic polycrystals with pre-existing cracks subjected to thermal loading problem is performed. Finally, a similar case is considered by investigating the thermo-mechanical behavior of a ceramic material made of two materials. The solution to the dynamic analysis is obtained by using explicit time integration and utilizing a stable time step size to obtain a stable solution as explained in [36].

All problem cases have two-dimensional models based on plane-stress assumption. The thickness of the plate is provided for the completeness of the information. The discretization size and horizon size used in numerical cases are sufficient to obtain accurate results with reasonable computational time. To demonstrate the suitability of the discretization and horizon size utilized in this study, convergence studies are performed and demonstrated in Appendix.

\subsection{Static analysis of isotropic material under thermal loading}

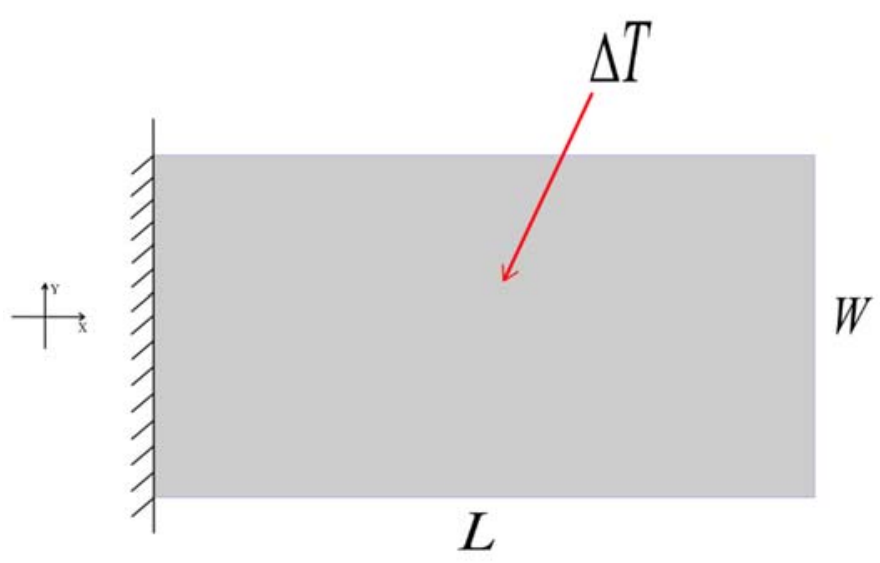

Fig. 4. Two-dimensional plate subjected to thermal loading.

The two-dimensional model shown in Fig. 4 has a length of $L=0.1542 \mathrm{~m}$ and a width of $W=0.0762 \mathrm{~m}$. The thickness of the plate is $0.1651 \times 10^{-3} \mathrm{~m}$. The elastic modulus and Poisson's ratio are $200 \mathrm{GPa}$ and $1 / 3$, respectively, and the density of the material is 7850 $\mathrm{Kg} / \mathrm{m}^{3}$. The thermal expansion coefficient is given as $23 \times 10^{-6} /{ }^{\circ} \mathrm{C}$. The PD model is generated by $240 \times 120$ points and the grid spacing is $d x=0.635 \times 10^{-3} \mathrm{~m}$. The horizon size is selected as $\delta=3 \times d x$. The left edge of the plate is fully constrained by creating a fictitious boundary region with a thickness equivalent to the horizon size. Both displacement components in $x$ - and $y$-directions are costrained inside the fictitious region. The thermal loading is applied in the form of a temperature change as $900^{\circ} \mathrm{C}$ for all nodes, i.e. $\Delta T=900^{\circ} \mathrm{C}$. The results of the points on the vertical and horizontal centerlines of the plate are chosen to compare with the 
FEM results and the variation of the displacements along the centerlines are shown in Fig. 5. It can be seen that the results of PD are identical to the FEM results and the accuracy of the model for the thermal expansion problem of isotropic material is verified.
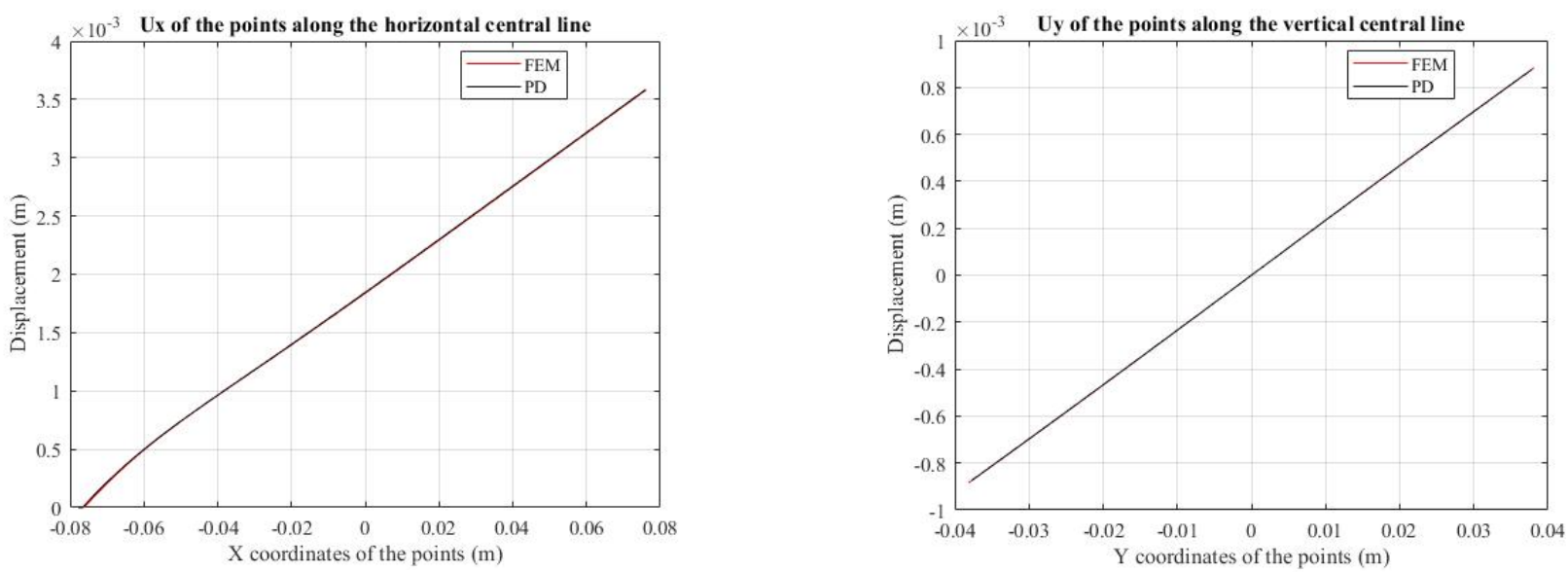

Fig. 5. Displacements of material points along the central lines of an isotropic material.

\subsection{Static analysis of a single cubic crystal under thermal loading}

For the second numerical case, a single cubic crystal is considered. The size of the model for the thermal single crystal analysis is the same as defined in Section 3.1. The material of the cubic crystal is iron and for iron crystal, the stiffness matrix coefficients are given as $c_{11}=231.4 \mathrm{GPa}, c_{12}=134.7 \mathrm{GPa}$, and $c_{44}=116.4 \mathrm{GPa}$. The orientation of the crystal is defined as $0^{\circ}$, i.e. Type- 2 bonds are placed at $45^{\circ}, 135^{\circ}, 225^{\circ}$, and $315^{\circ}$ orientations. The loading condition and boundary condition are also the same as in the previous section. The PD displacements, in $x$ - and $y$ - directions, of the two orthogonal centerlines are collected and their comparison against FEM is given in Fig. 6. Results from both approaches are in good agreement with each other which shows that the microscale PD model can provide accurate results for the thermal loading problems of a cubic crystal under static loading condition.
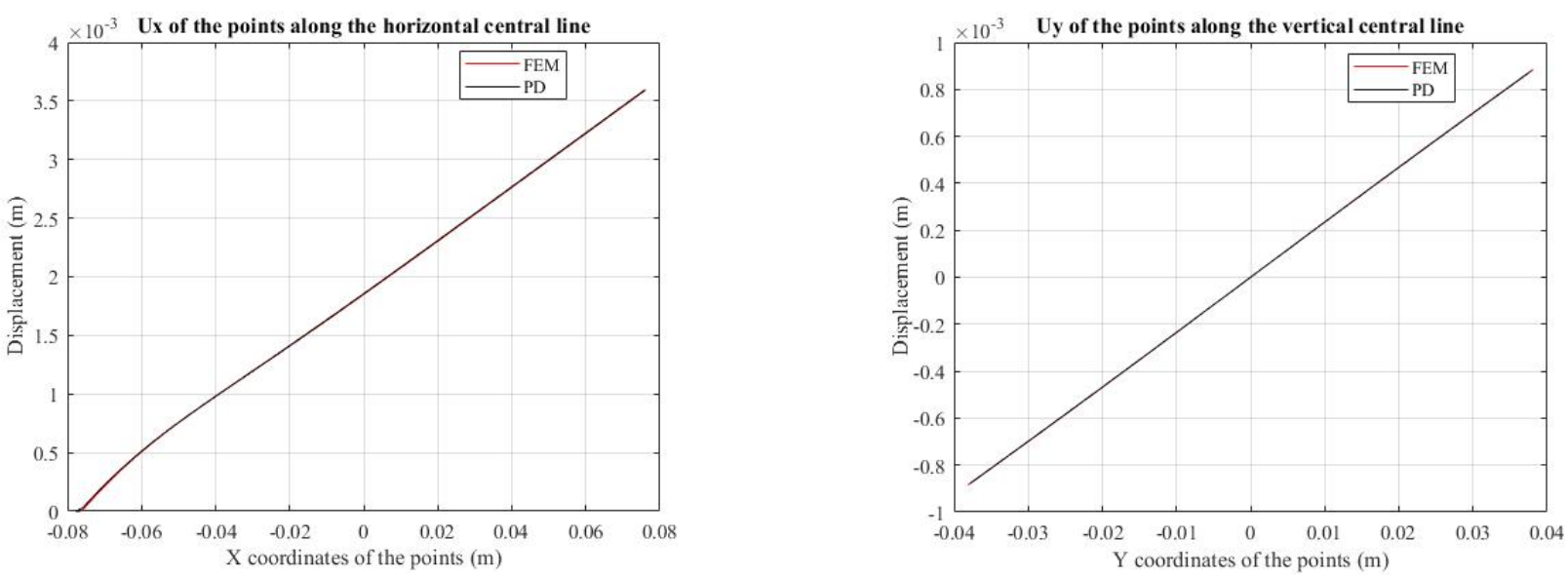

Fig. 6. Displacements of material points along the central lines of a single cubic crystal. 


\subsection{Static analysis of cubic polycrystals under thermal loading}

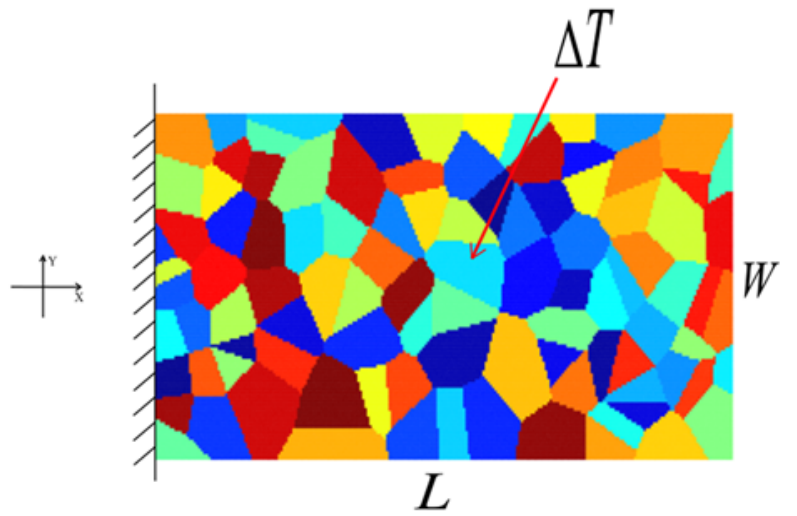

Fig. 7. Polycrystalline structure subjected to thermal loading.

In order to further test the accuracy of the model for cubic polycrystals, a $5 \mathrm{~mm} \times 2.5 \mathrm{~mm}$ iron plate with a thickness of $0.5 \mathrm{~mm}$ is considered with 100 grains (Fig. 7). Apart from the change in the geometry of the plate, the remaining parameters are the same as in the single-crystal example. For each grain, the orientation of the crystal is randomly selected. By comparing the displacements between PD and FEM results as shown in Fig. 8, it can be concluded that for cubic polycrystals, the PD model can capture correct results for the thermally-induced deformation problem.
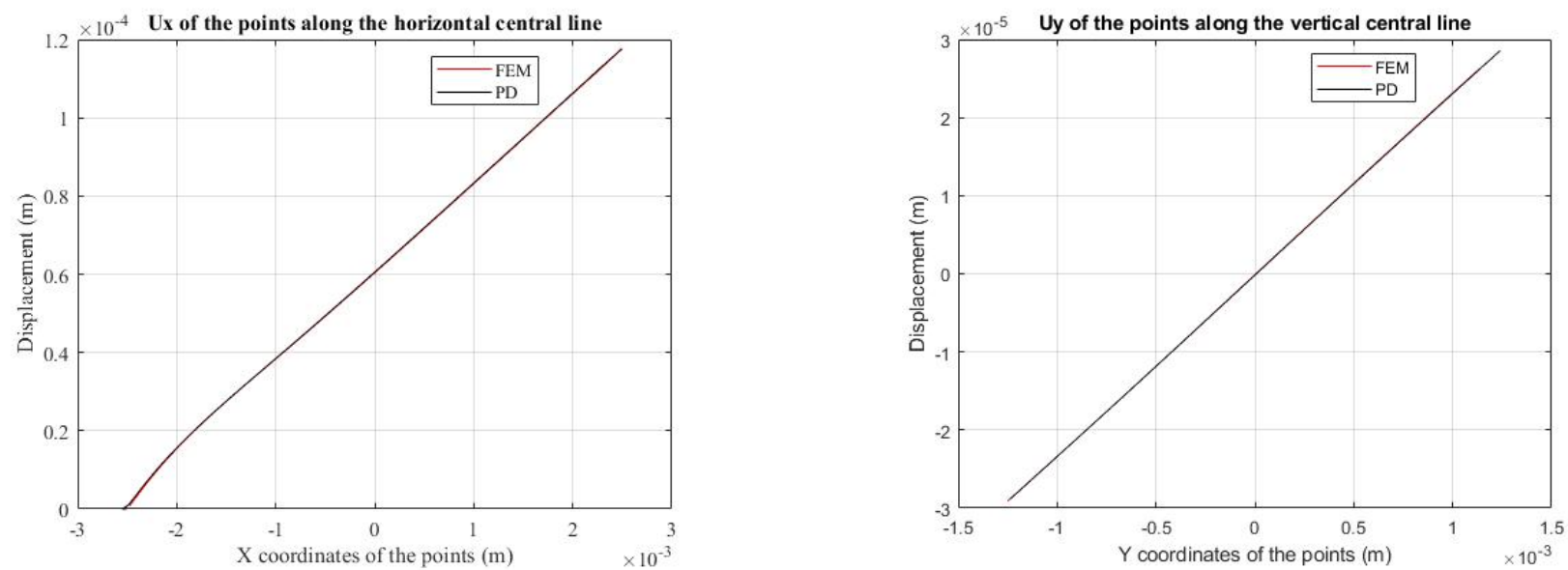

Fig. 8. Displacements of the material points along the central lines of cubic polycrystals.

\subsection{Dynamic analysis of cubic polycrystals with pre-existing cracks under thermal loading}

For the purpose of investigating the fracture behavior and the effects of grain size and grain boundary strength for the cubic polycrystalline materials under thermal loading, a $10 \mathrm{~mm} \times 10 \mathrm{~mm}$ plate made of $\beta$-silicon carbide was chosen and the model was created using $200 \times 200$ PD points. The thickness of the plate is the same with the PD grid size $\mathrm{dx}$ which is equal to $0.05 \mathrm{~mm}$. The stiffness matrix coefficients for the silicon carbide are given as $\mathrm{c}_{11}=395 \mathrm{GPa}, \mathrm{c}_{12}=123 \mathrm{GPa}$, and $\mathrm{c}_{44}=236 \mathrm{GPa}$ [38]. The density of the silicon carbide is $3100 \mathrm{Kg} / \mathrm{m}^{3}$ and the thermal expansion coefficient is $6.58 \times 10^{-6} /{ }^{\circ} \mathrm{C}$. The critical stretch of the silicon carbide was calculated using the fracture toughness of $3.46 \times 10^{6} \mathrm{MPa} \sqrt{\mathrm{m}}$ [39]. The time step size for explicit time integration is specified as $1 \times 10^{-9} \mathrm{~s}$. For the dynamic cooling 
process, the model is free from any mechanical constraints and the temperature decreases by $300{ }^{\circ} \mathrm{C}$, i.e. $\Delta T=-300^{\circ} \mathrm{C}$, over a short period of time $\left(3 \times 10^{-6} \mathrm{~s}\right)$. Two different types of preexisting cracks were considered. The first one is a $2 \mathrm{~mm}$ crack horizontally located at the center of the plate. For the second type, two small cracks were located at the top and bottom edges of the plate with a length of $1 \mathrm{~mm}$ (Fig. 9).
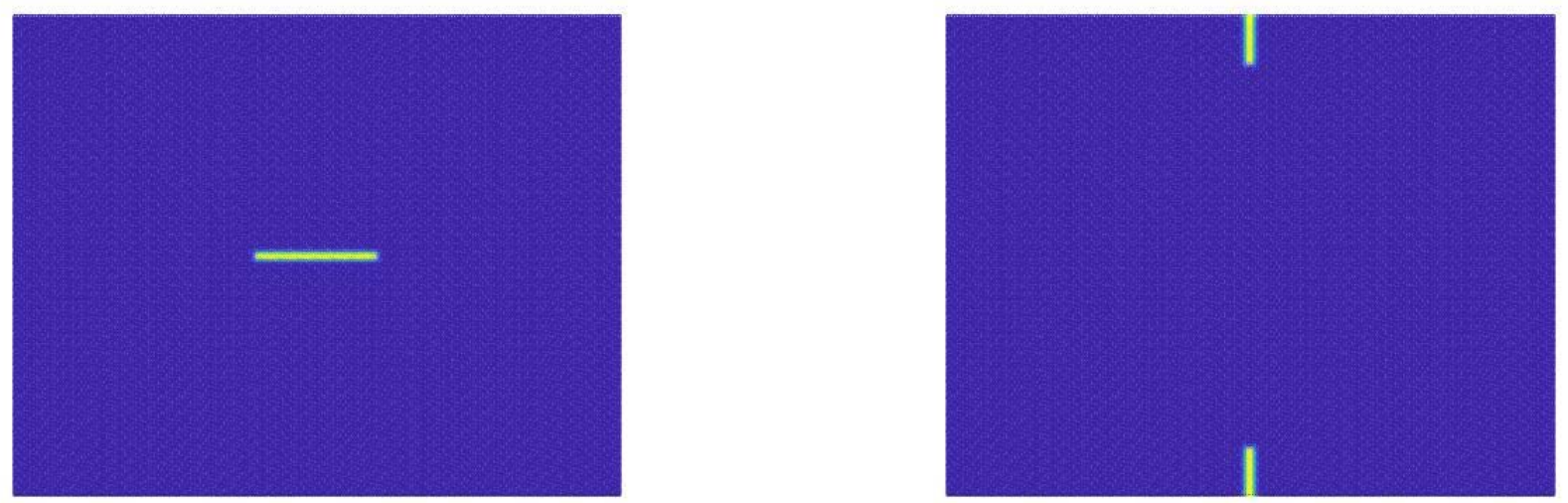

Fig. 9. Pre-existing cracks (a) horizontal, (b) vertical.

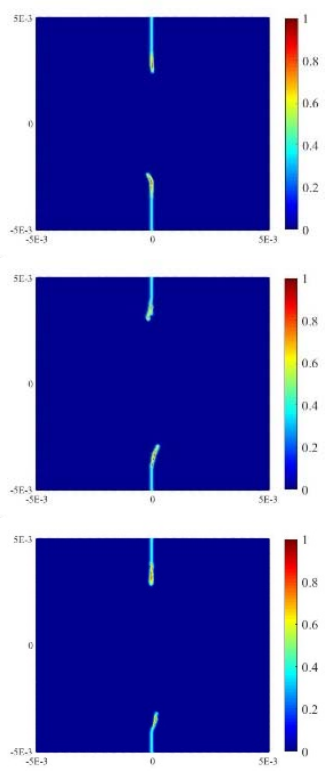

$0.75 \mu \mathrm{s}$
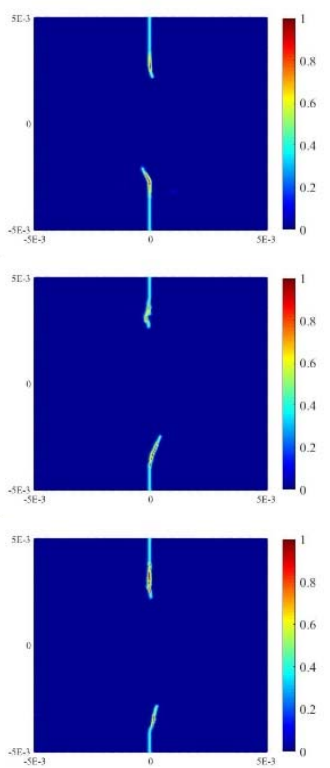

$1.5 \mu \mathrm{s}$
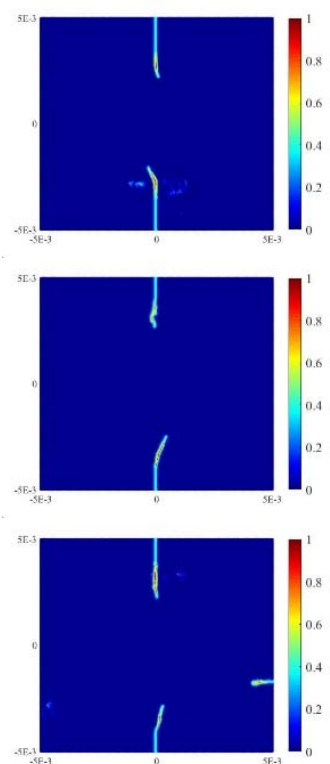

$2.25 \mu \mathrm{s}$
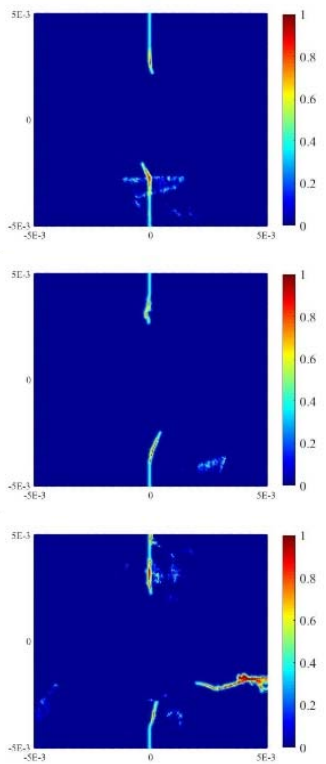

$3 \mu s$

Fig. 10. Crack propagation for the vertical pre-existing crack case for different number of grains. From the top to the bottom the number of the grains are: 20, 50, and 100 .
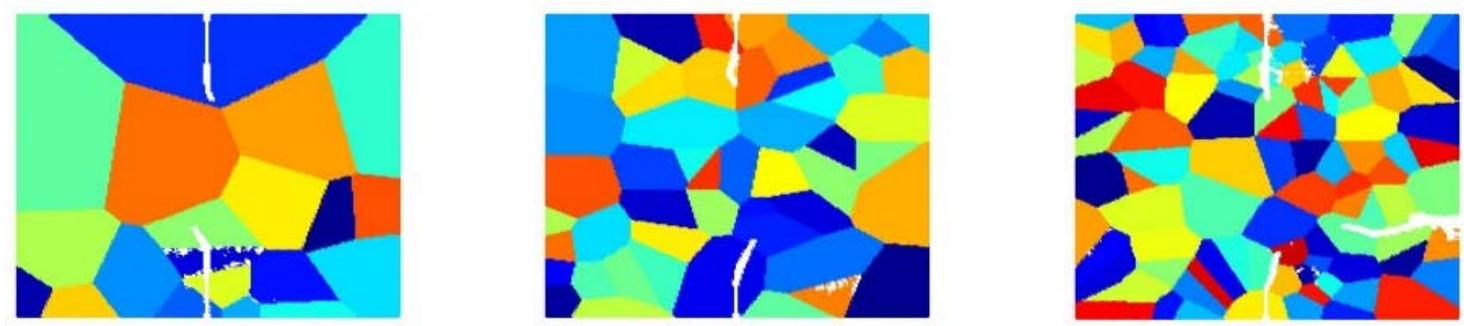

Fig. 11. Crack propagation for the vertical pre-existing crack case at $3 \mu$ s for 20,50 , and 100 grains. 
The plate was created using a different number of grains $(20,50$, and 100$)$ to explore the effect of the grain size on fracture behavior. Grain Boundary Coefficient is specified as GBC $=1$. The plots of the propagation of the cracks are shown in Figs. 10 and 12 and the crack propagation inside the crystals are also presented in Figs. 11 and 13. For the vertical crack, with the decrease of the temperature and shrinkage of the plate, the crack starts to propagate from the initial notch. However, it can be seen that the speed of the crack propagation is slowing down and there is no significant crack propagation after $1.5 \mu \mathrm{s}$. During the entire process, there is no obvious crack branch occurance. Based on the plots of the final stage, the number of grains do not have a big impact on the main crack. However, for the 100 grains plate, there is an initiation of a new crack from the boundary of the plate. On the basis of the crystal plots with crack, all of the three cases are showing transgranular crack pattern and the cracks propagate through the crystals rather than along the crystal boundaries. The crack nucleation at the right edge, for the 100 grains case, is mainly caused due to increase in grain boundaries as the number of grains increase.
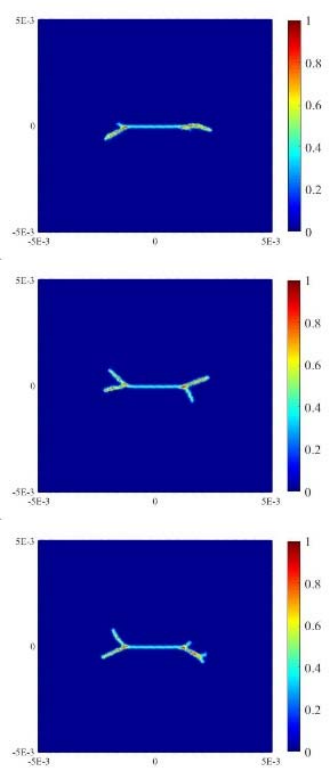

$0.75 \mu \mathrm{s}$
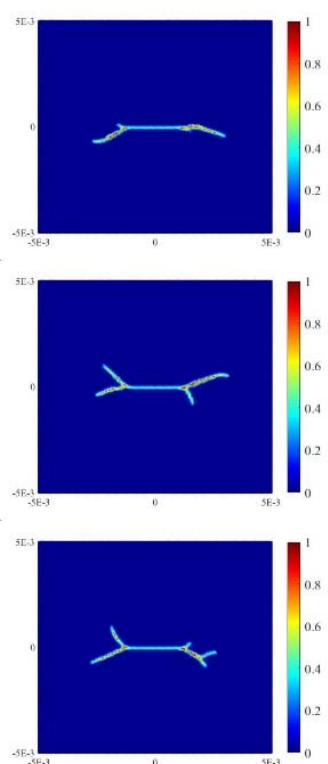

$1.5 \mu \mathrm{s}$
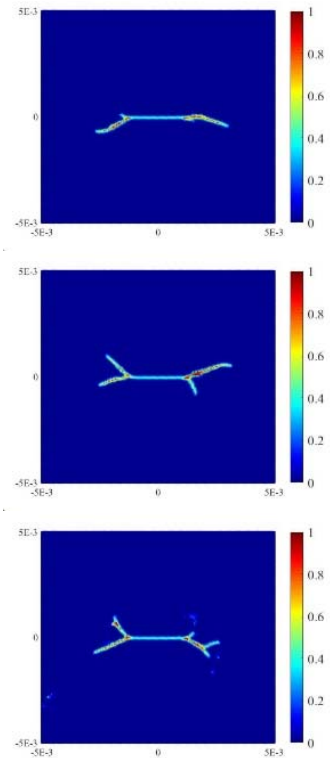

$2.25 \mu \mathrm{s}$
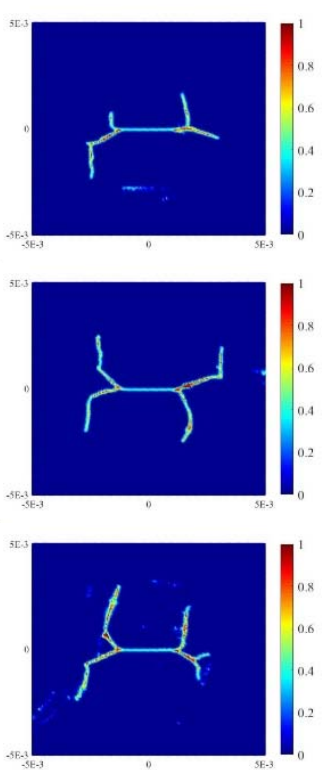

$3 \mu s$

Fig. 12. Crack propagation for the horizontal pre-existing crack case for different number of grains. From the top to the bottom the number of the grains are: 20, 50, and 100.
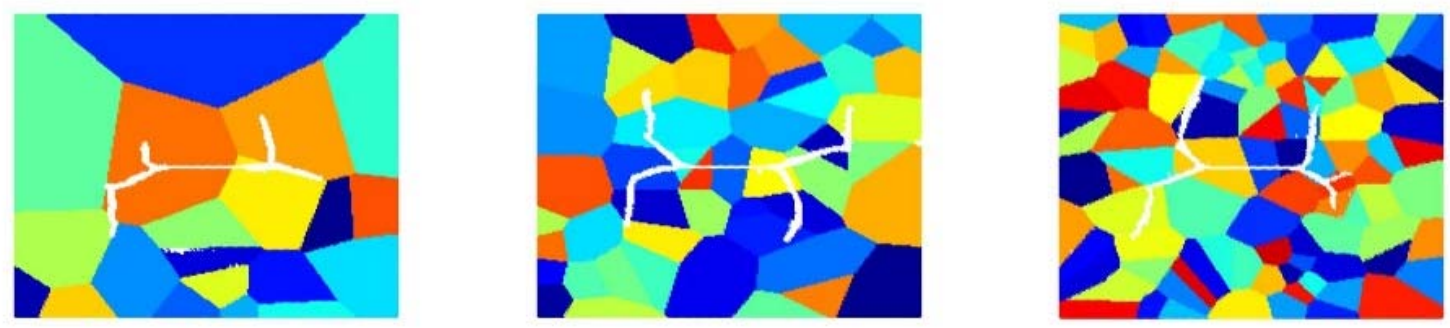

Fig. 13. Crack propagation for the horizontal pre-existing crack case at $3 \mu$ s for 20,50 , and 100 grains.

For the horizontal pre-existing crack case, with the shrinkage of the plate, the crack branches to propagate but the propagation is not severe until the temperature change is over $225^{\circ} \mathrm{C}$ and then the crack propagates dramatically from the branch tip. It can be seen that the plate with 
100 grains has more branches and the boundary has more potential to initiate new cracks than the remaining two cases. This can also be explained by the increase of grain boundaries similar to the vertical crack condition. It can be concluded that with the increase of number of grains, the possibility of new crack initiation increases. Moreover, at the final stage, even if the crack path is not the same, the fracture severity of three cases are still in a similar manner. This means that the patterns of the main crack are not significantly influenced by the number of grains, which are also in accordance with the conclusion of De Meo et al. [1]
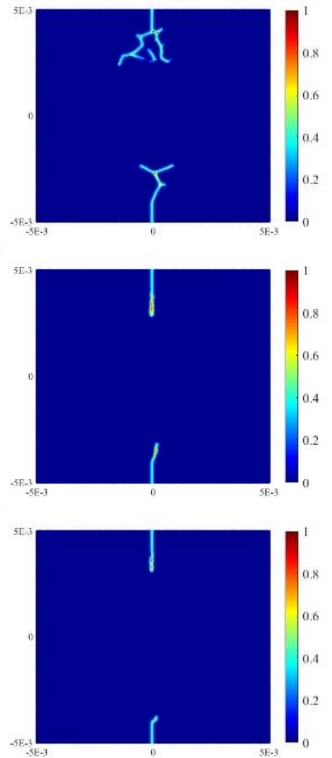

$0.75 \mu \mathrm{s}$
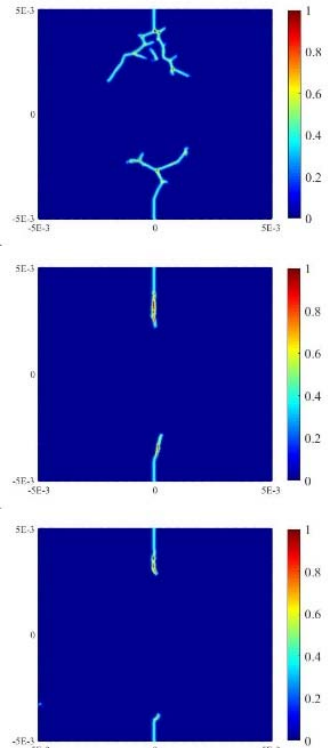

$1.5 \mu \mathrm{s}$
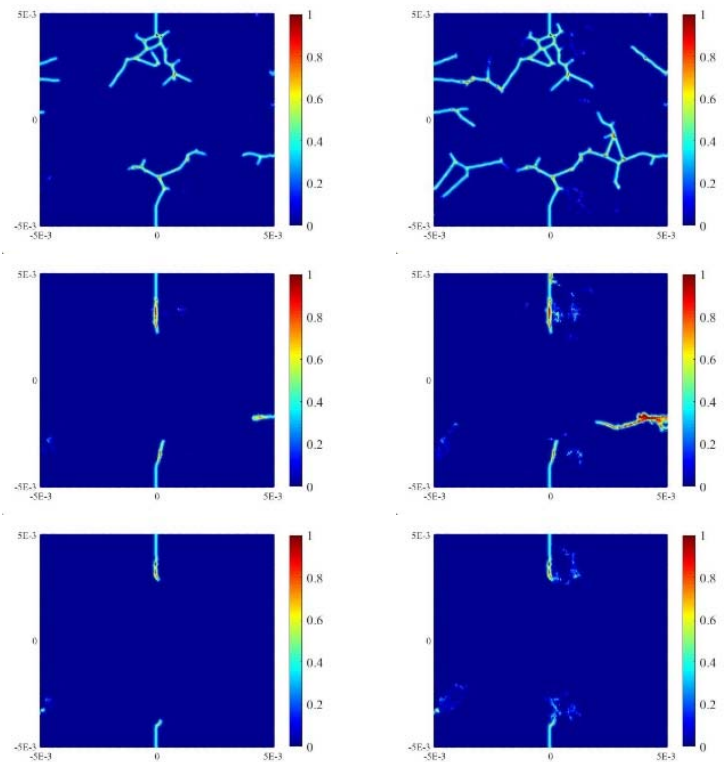

$2.25 \mu \mathrm{s}$

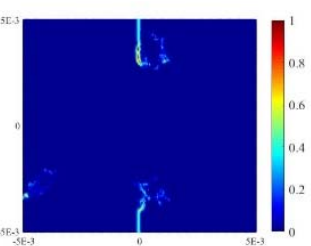

$3 \mu \mathrm{s}$

Fig. 14. Crack propagation for the vertical pre-existing crack for different values of GBC.

From the top to the bottom the values of GBC are: $0.5,1$, and 2.

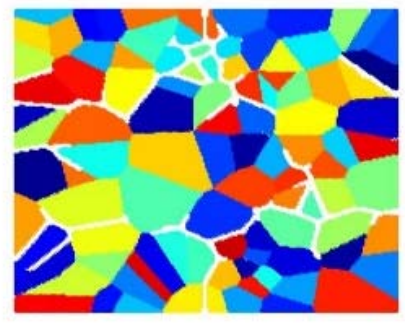

(a)

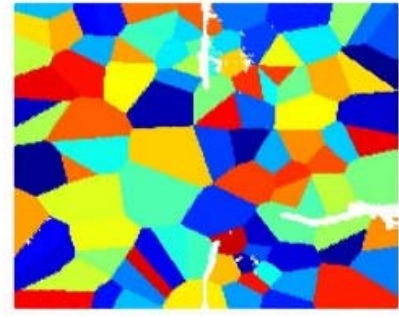

(b)

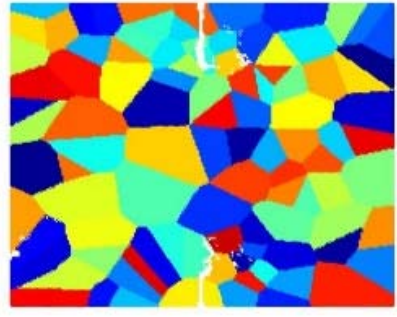

(c)

Fig. 15. Crack propagation for the vertical pre-existing crack at $3 \mu$ s for GBC values of (a) 0.5 , (b) 1 , and (c) 2 .

Next, different values of the GBC are considered for 100 grains and both types of cracks to investigate the effect of grain boundary strength. It is expected that if the GBC is less than 1, the fracture behavior becomes more intergranular because the strength of the bond crossing the grain boundary becomes weaker than the bonds inside the grains. On the contrary, the crack pattern becomes transgranular with the growth of the value of GBC and the crack prefers to propagate within the crystals rather than along the grain boundaries. As shown in Figs. 14-17, 
Regardless for the crack being vertical or horizontal, when $\mathrm{GBC}=0.5$, the crack propagates from the pre-existing crack and the newly generated crack becomes much more severe than the remaining two cases. Additionally, it can be seen from the plots of the grains that the crack starts to propagate along the grain boundaries when $\mathrm{GBC}=0.5$. It should also be mentioned that the crack patterns are not strongly influenced when $\mathrm{GBC}=2$ with respect to $\mathrm{GBC}=1$ and the plots for these two cases are roughly identical except that there are new cracks initiated from the grain boundaries for the vertical crack condition for $\mathrm{GBC}=1$. Actually, this phenomenon also meets the observation of De Meo et al. [1] and it can be inferred that the strong grain boundaries will be beneficial for reducing the possibility of initiation of new cracks.
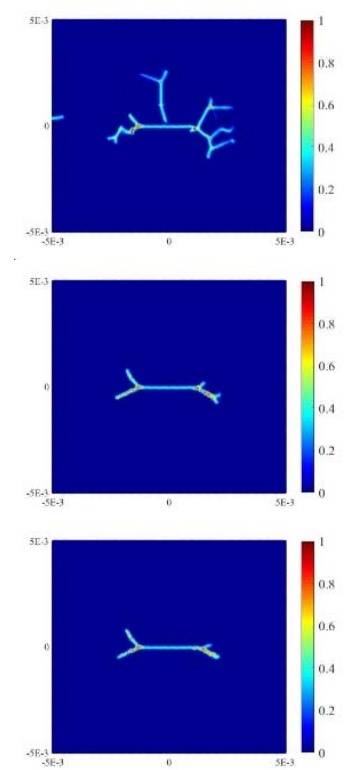

$0.75 \mu \mathrm{s}$
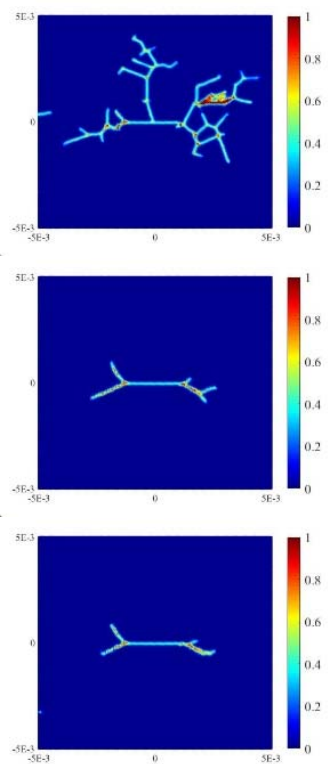

$1.5 \mu \mathrm{s}$
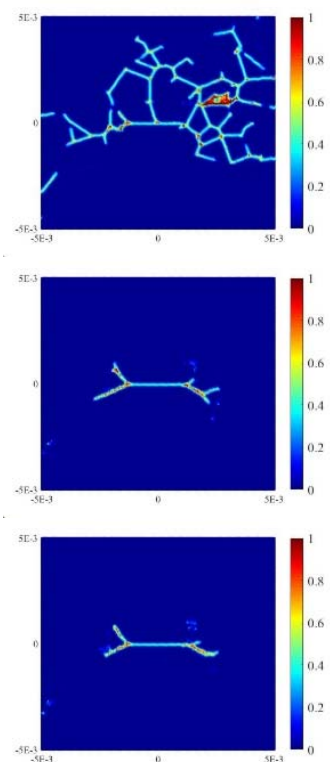

$2.25 \mu \mathrm{s}$
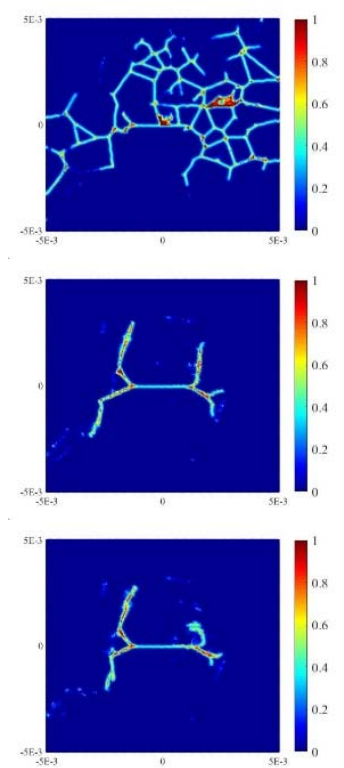

$3 \mu \mathrm{s}$

Fig. 16. Crack propagation for the horizontal pre-existing crack case for different values of GBC. From the top to the bottom the values of GBC are: $0.5,1$, and 2

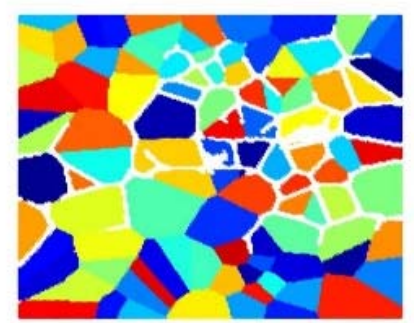

(a)

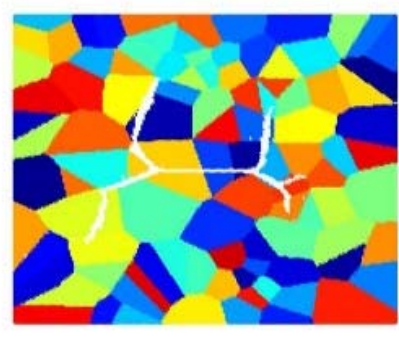

(b)

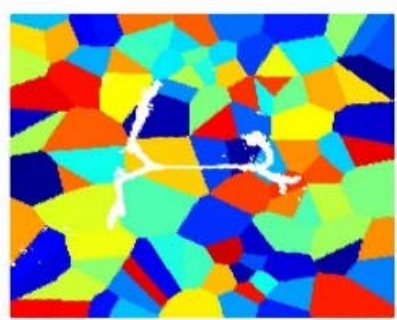

(c)

Fig. 17. Crack propagation for the horizontal pre-existing crack case at $3 \mu \mathrm{s}$ for GBC values of (a) 0.5 , (b) 1 , and (c) 2 .

3.5 Dynamic analysis of ceramic made of two materials with pre-existing cracks under thermal loading

In this section a type of ceramic, which is constituted of two materials (silicon carbide and alumina), is considered under thermal loading condition based on an earlier study by Geraci 
and Aliabadi [17]. They utilize the BZM to investigate the effect of number of grains and material composition (the percentages of the silicon carbide). In this study, the PD model is used to analyze the same problem and the effect of the GBC is also taken into consideration. Although the crystal system for silicon carbide and alumina are cubic/hexagonal and trigonal, respectively, according to [17], these two materials can be treated as isotropic materials for simplification. To be able to compare PD results with the results given in [17], same approach is utilized by treating two materials as isotropic materials. The required parameters for the two materials are collected and summarized in Table 1 [17]:

\begin{tabular}{|c|c|c|c|c|}
\hline Materials & $\begin{array}{c}\text { Elastic Modulus } \\
(\mathrm{GPa})\end{array}$ & $\begin{array}{c}\text { Thermal Expansion } \\
\text { Coefficient }\left(/{ }^{\circ} \mathrm{C}\right)\end{array}$ & $\begin{array}{c}\text { Density } \\
\left(\mathrm{Kg} / \mathrm{m}^{3}\right)\end{array}$ & $\begin{array}{c}\text { Fracture toughness } \\
(\mathrm{MPa} \sqrt{\mathrm{m}})\end{array}$ \\
\hline Silicon carbide & 470 & $4 \times 10^{-6}$ & 3100 & 3 \\
\hline Alumina & 431 & $8.4 \times 10^{-6}$ & 3690 & 3 \\
\hline \multicolumn{4}{|c|}{ Table 1. Material information for silicon carbide and alumina } \\
\hline
\end{tabular}
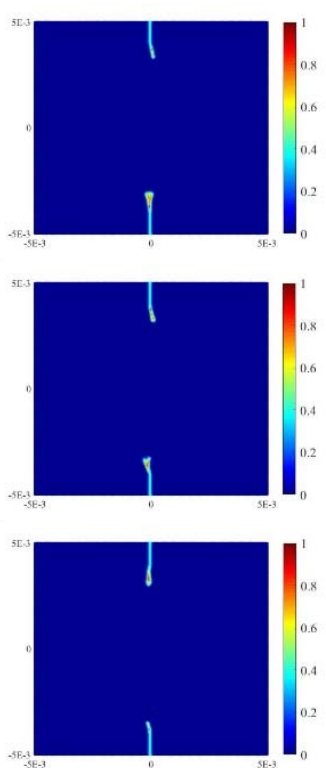

$0.5 \mu \mathrm{s}$
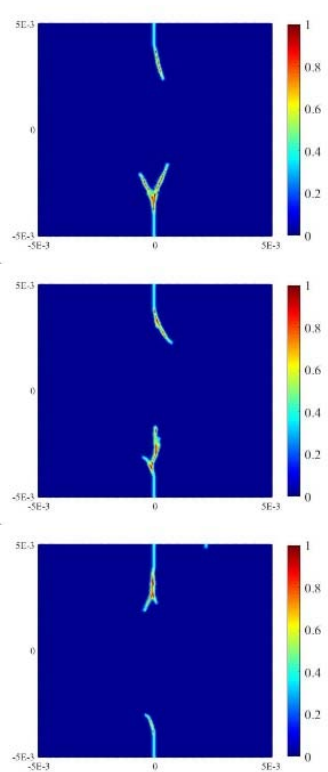

$1 \mu s$
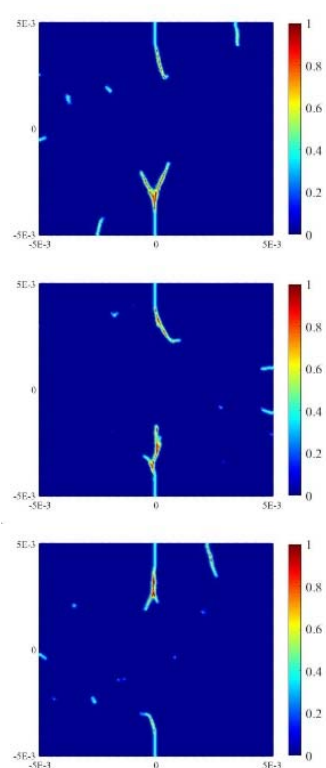

$1.5 \mu \mathrm{s}$
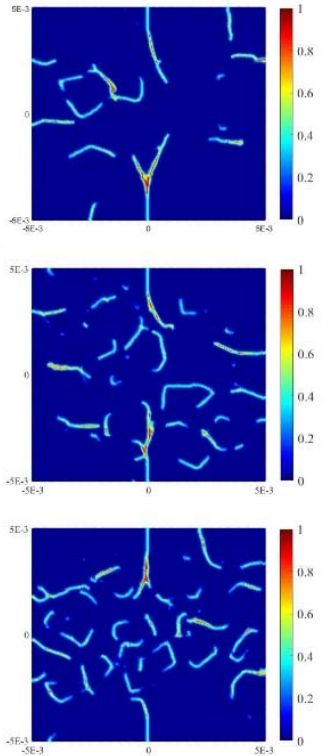

$2 \mu \mathrm{s}$

Fig. 18. Crack propagation for the vertical pre-existing crack case for different number of grains with $50 \%$ silicon carbide. From the top to the bottom the number of the grains are: 20,50 , and 100 . 

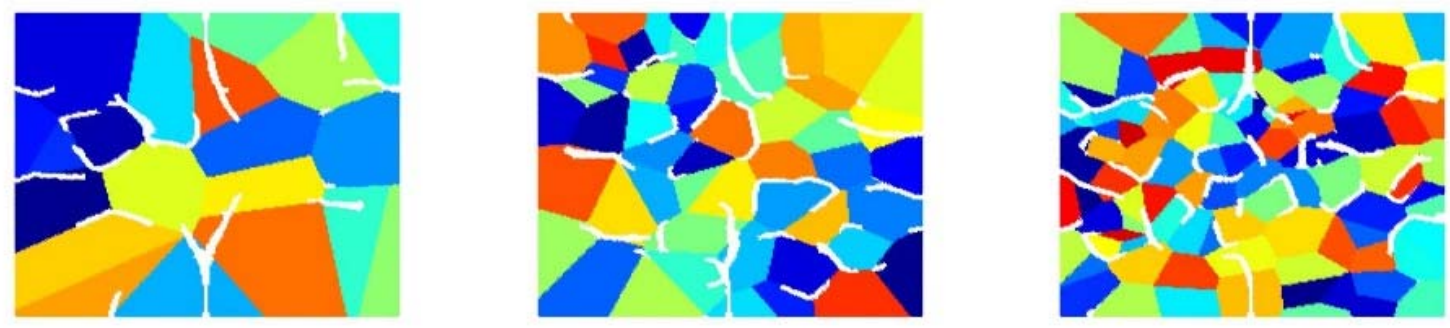

(a) Crack propagation showing the distribution of crystals.
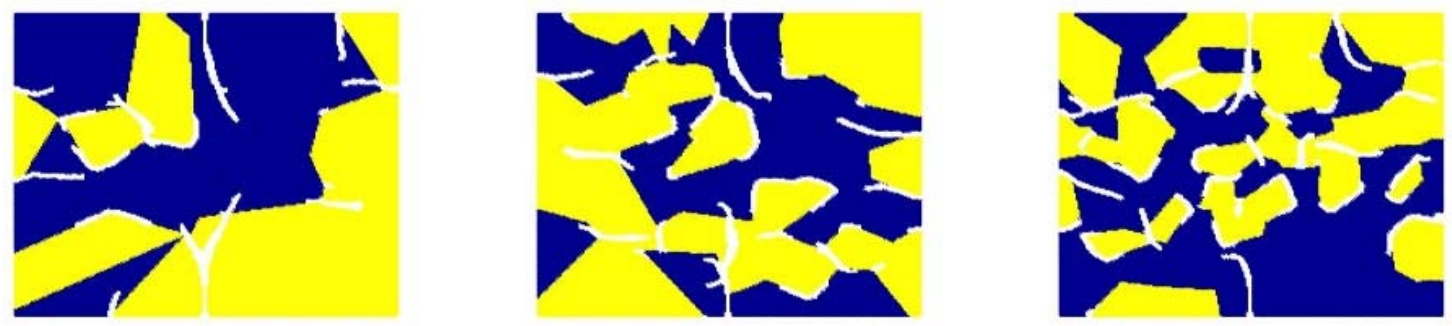

(b) Crack propagation showing the material composition (blue represents silicon carbide)

Fig. 19. Crack propagation for the vertical pre-existing crack case with crystals and material composition at $2 \mu \mathrm{s}$ for 20,50 , and 100 grains with $50 \%$ silicon carbide.

The geometry of the model (number of PD points, the patterns of the pre-existing cracks, and the size of the plate) is same as the previous Section 3.4. The only difference is the range of temperature change which has been reduced to $200{ }^{\circ} \mathrm{C}$ because the currently used materials are more sensitive to the thermal loading. For the condition of different number of grains, 20, 50 , and 100 grains are considered with $\mathrm{GBC}=1$. The percentage of the silicon carbide is kept as $50 \%$, i.e. almost half of the grains are made of silicon carbide and the rest is alumina. As shown in Fig. 18, for the vertical crack, with the decrease of the amount of the grains, the main crack becomes easier to branch and the crack is more likely to propagate approaching the other edge of the plate. These results agree with the conclusion drawn by Geraci and Aliabadi [17]. The damage plots with the distribution of crystals and the two materials are also provided in Fig. 19 to support the conclusion. 

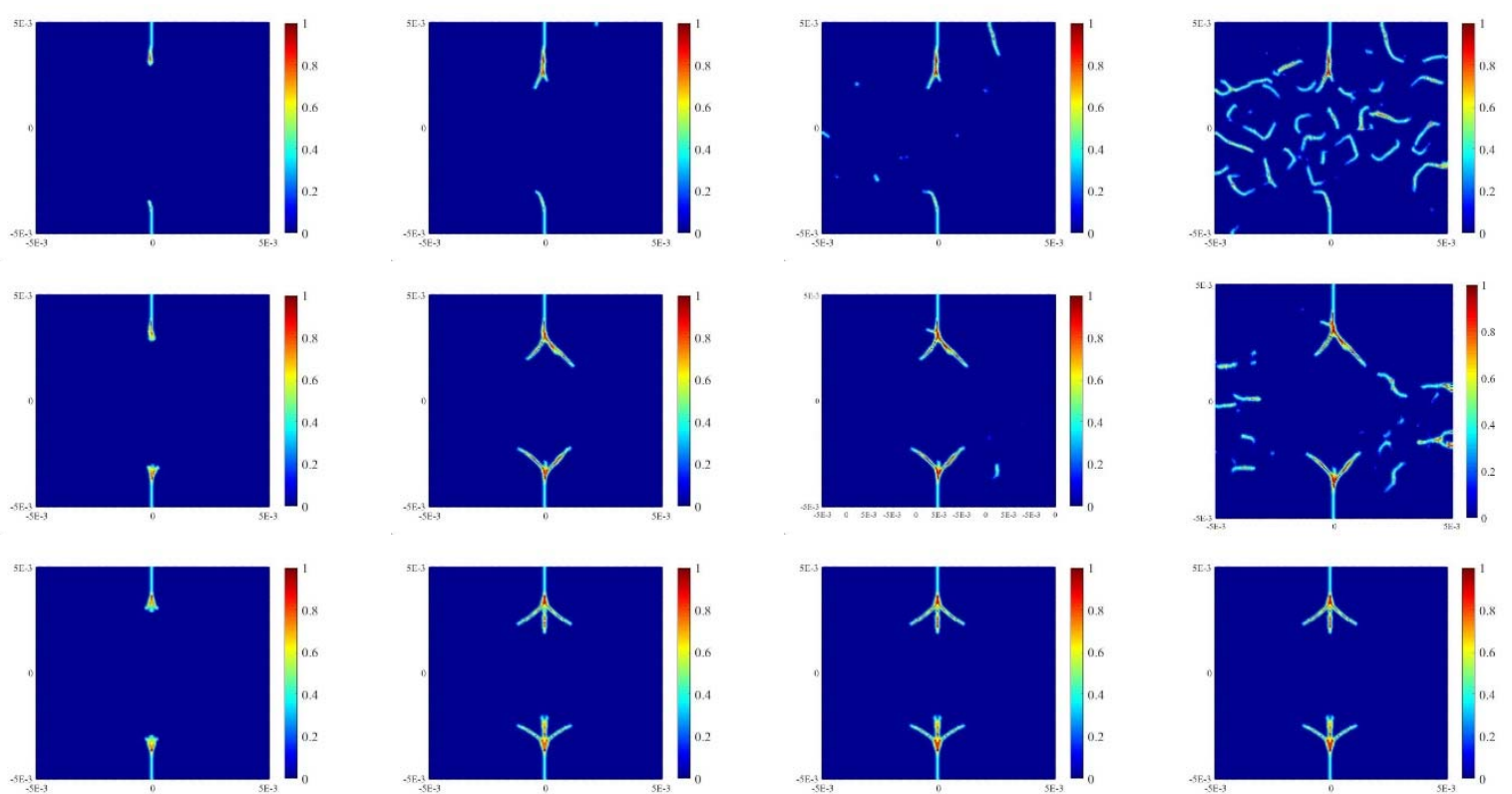

$0.5 \mu \mathrm{s}$

$1 \mu \mathrm{s}$

$1.5 \mu \mathrm{s}$

$2 \mu \mathrm{s}$

Fig. 20. Crack propagation for the vertical pre-existing crack for different percentages of silicon carbide with 100 grains. From the top to the bottom the percentages of the silicon carbide are: $50 \%, 10 \%$, and $0 \%$
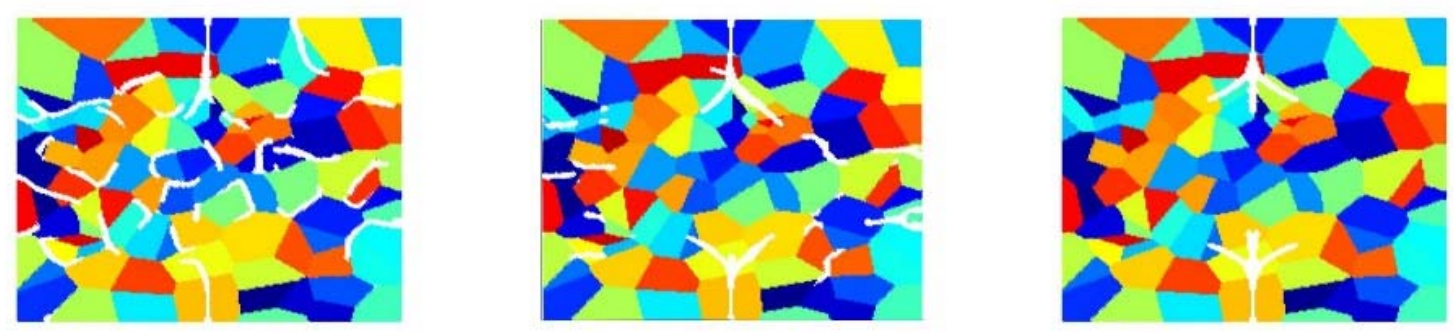

(a) Crack propagation showing the distribution of crystals.
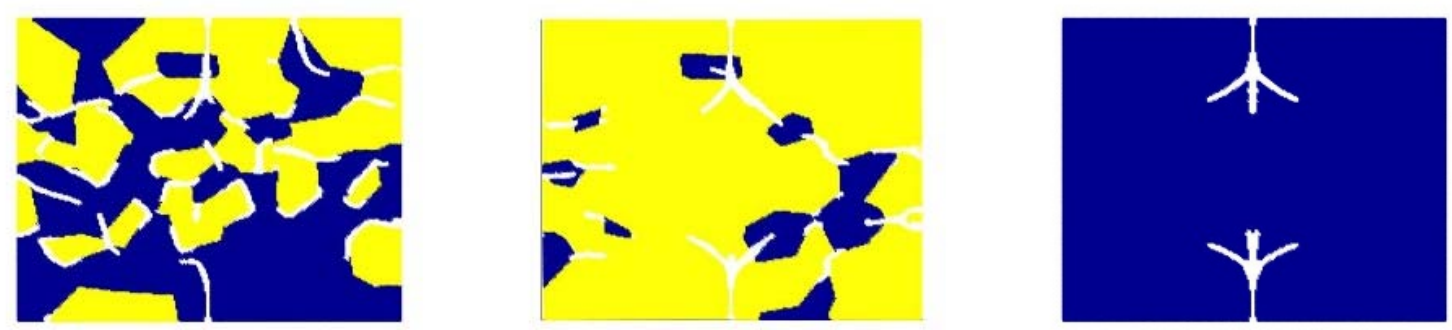

(b) Crack propagation showing the material composition (blue represents silicon carbide).

Fig. 21. Crack propagation for the vertical pre-existing crack case with crystals and material composition at $2 \mu \mathrm{s}$ for $50 \%, 10 \%$, and $0 \%$ silicon carbide with 100 grains.

In order to test the influence of the percent of silicon carbide, the plate with 100 grains is considered. The number of silicon carbide crystals was reduced from $50 \%$ to $10 \%$ and then to $0 \%$. Due to the fact that the thermal expansion coefficient of silicon carbide is about half of the alumina and the strength and fracture toughness of the two materials are quite close, it is much easier for the crack to propagate in alumina rather than in silicon carbide. As can be seen 
from the plots in Fig. 20, the main crack is branching although this is not obvious for 50\% silicon carbide case. However, when the percentage has been reduced to $10 \%$, there exist two branches at the initial crack tip and the number of branched cracks increases to 3 when the material is pure alumina. It can be concluded that with the increase of silicon carbide, the severity of the main crack is limited. However, the drawback is also prominent, there are small cracks initiating from the grain boundaries which can be further observed from Fig. 21. It can also be shown that the difference of coefficient of thermal expansion, $\alpha$, between different materials will lead to initiation of new cracks.

For the plate with 100 grains and 50\% silicon carbide, different GBC is also considered. As shown in Figs. 22 and 23, cracks initiating from the grain boundaries become much more severe with $\mathrm{GBC}=0.5$ and the shape of the single crystals made of different materials can be seen clearly. Furthermore, the crack patterns of the remaining two conditions are quite similar, which can also further enhance the conclusion about the effect of GBC in Section 3.4.
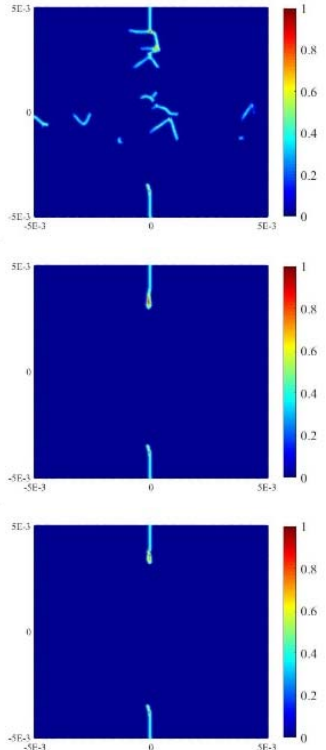

$0.5 \mu \mathrm{s}$
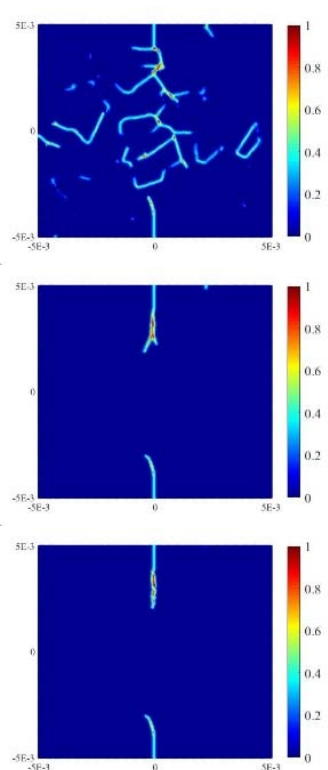

$1 \mu s$
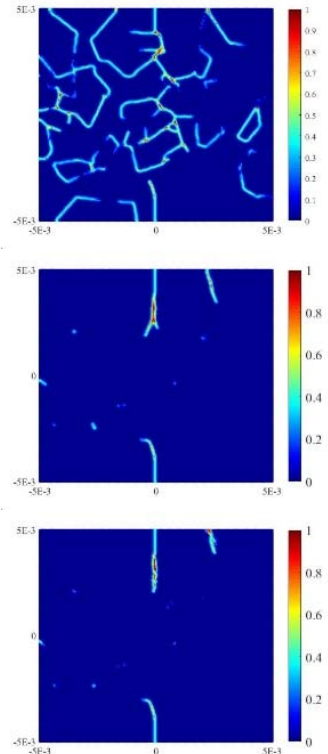

$1.5 \mu \mathrm{s}$
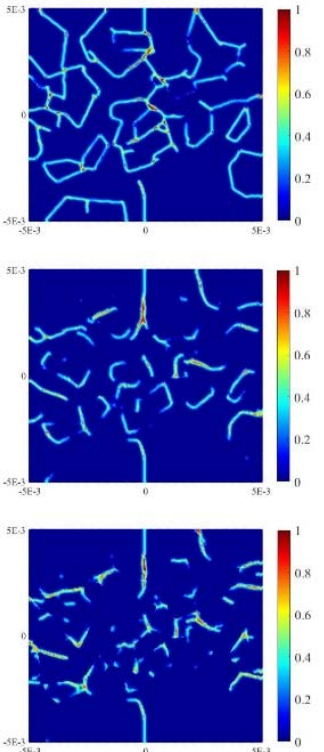

$2 \mu s$

Fig. 22. Crack propagation for the vertical pre-existing crack case for different values of GBC with 100 grains and 50\% silicon carbide. From the top to the bottom the values of GBC are: $0.5,1$, and 2 . 

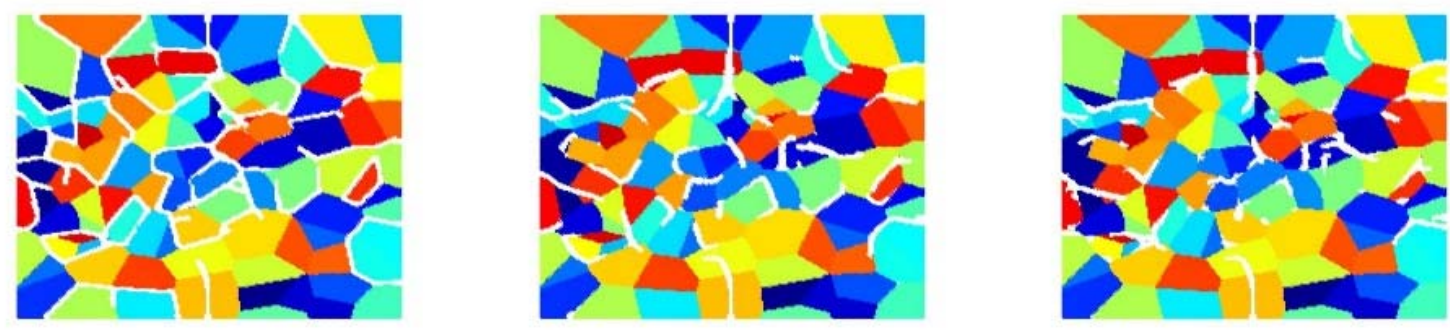

(a) Crack propagation showing the distribution of crystals.
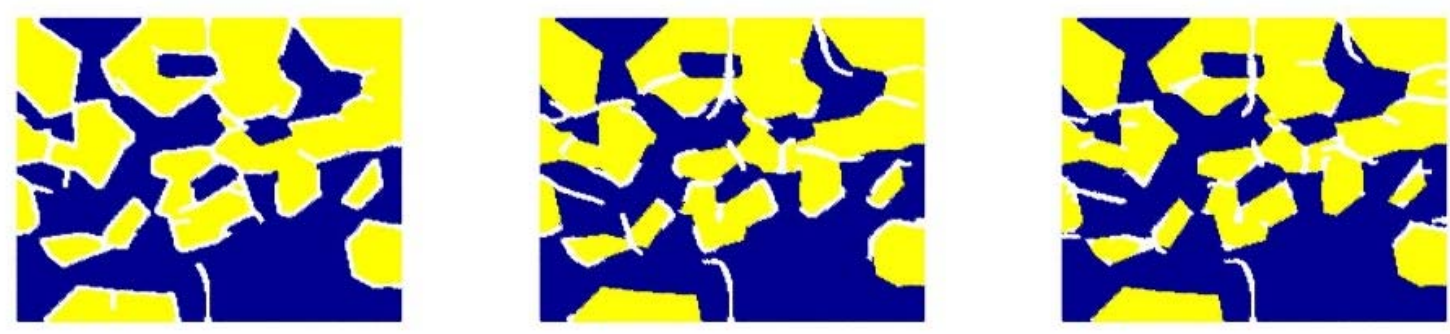

(b) Crack propagation showing the material composition (blue represents silicon carbide).

Fig. 23. Crack propagation for the vertical pre-existing crack case with crystals and material composition at $2 \mu \mathrm{s}$ for $0.5,1$, and $2 \mathrm{GBC}$ with 100 grains.

Horizontal pre-existing crack is also considered. After comparing the plots for various conditions (Fig. 24 to Fig. 29), the same conclusions can be obtained as the vertical pre-existing crack and they can be summarized as: 1) with the increase of number of grains, the propagation of the main crack and its branches become relatively hindered but the initiation of small cracks at the grain boundary become serious, 2) with the increase of silicon carbide, the severity of the main crack decreases due to the decrease of the branches, 3 ) if the grain boundary strength becomes weaker, the crack pattern becomes intergranular but increasing the value of GBC does not significantly influence the results. These conclusions are immune to the type of the preexisting crack and match well with Geraci and Aliabadi [17]. 

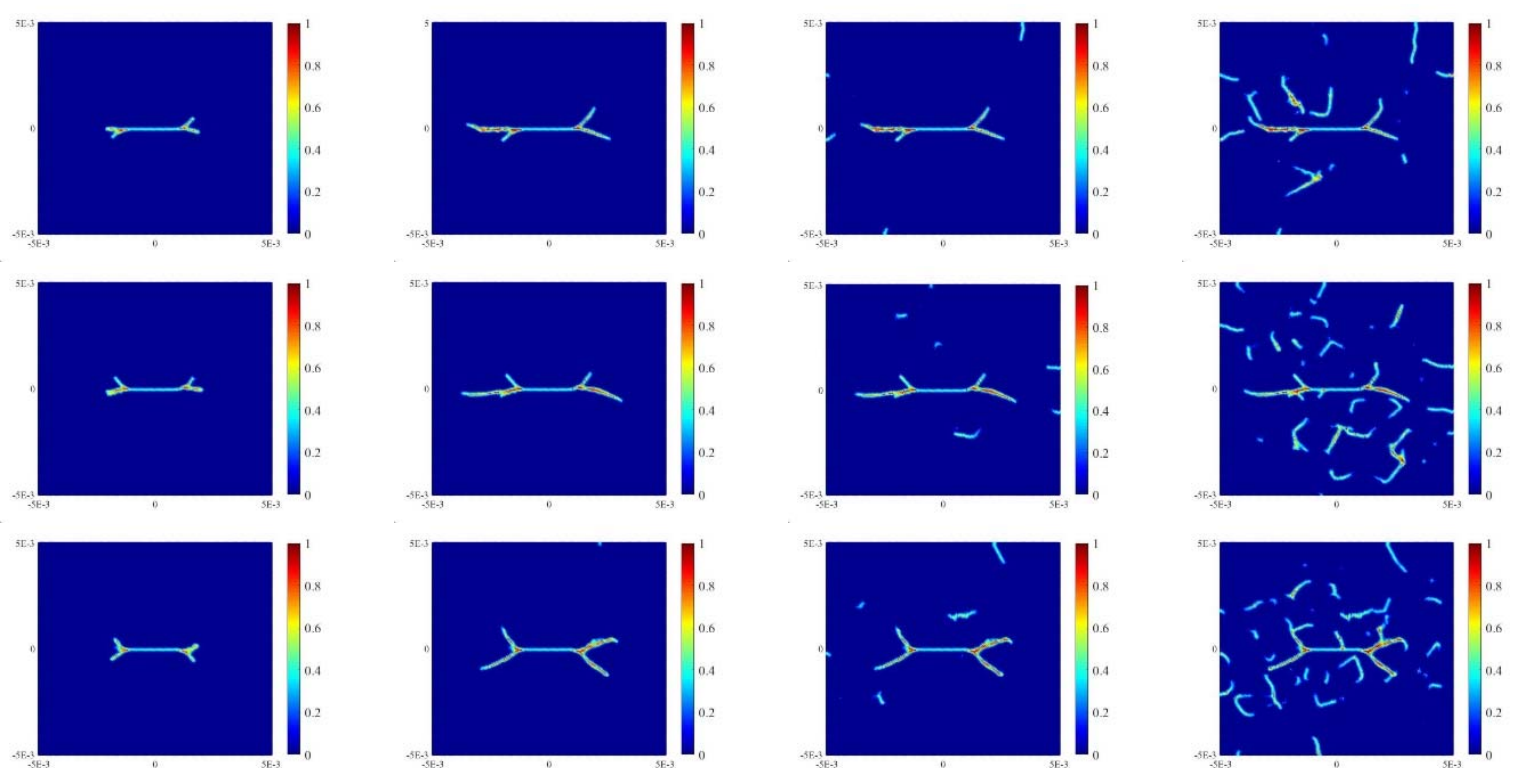

$0.5 \mu \mathrm{s}$

$1 \mu s$

$1.5 \mu \mathrm{s}$

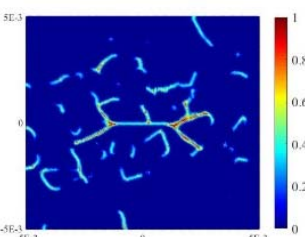

$2 \mu s$

Fig. 24. Crack propagation for the horizontal pre-existing crack case for different number of grains with $50 \%$ silicon carbide. From the top to the bottom the number of the grains are: 20,50 , and 100
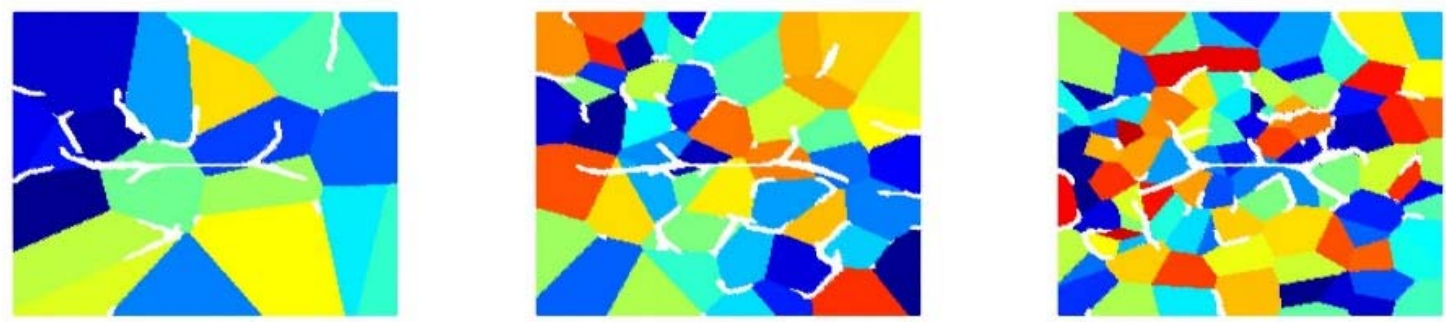

(a) Crack propagation showing the distribution of crystals.
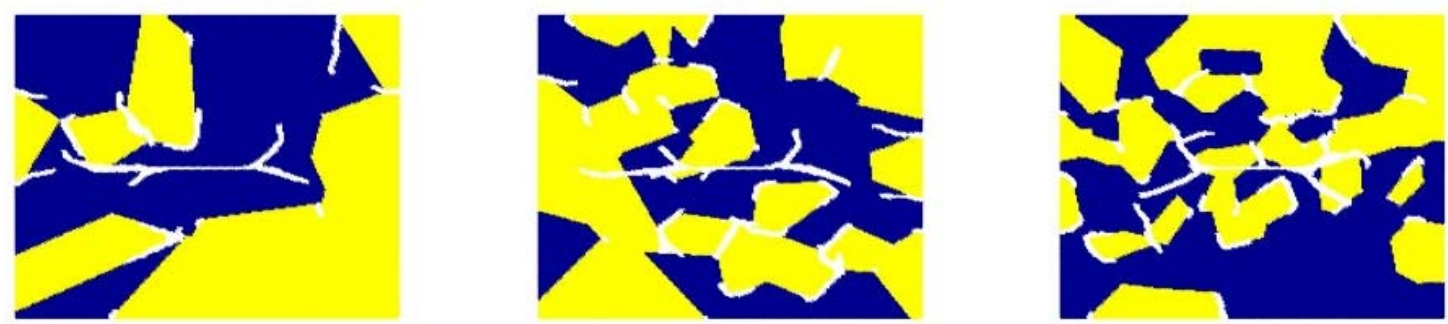

(b) Crack propagation showing the material composition (blue represents silicon carbide).

Fig. 25. Crack propagation for the horizontal pre-existing crack case with crystals and material composition at $2 \mu \mathrm{s}$ for 20,50 , and 100 grains with $50 \%$ silicon carbide. 

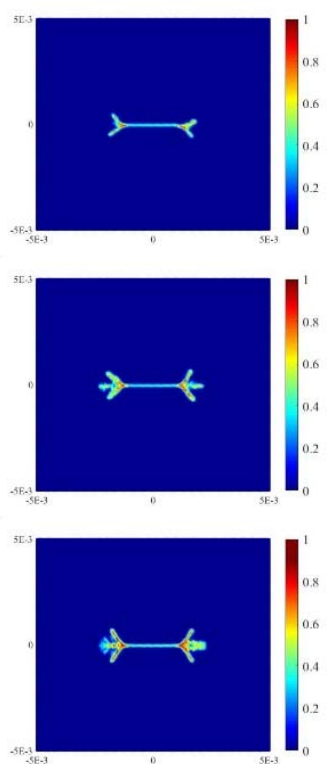

$0.5 \mu \mathrm{s}$
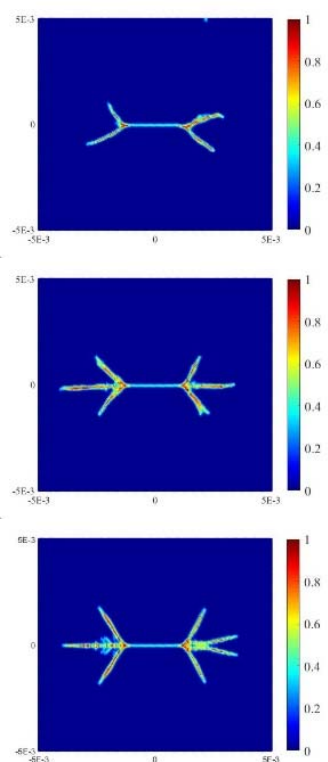

$1 \mu \mathrm{s}$
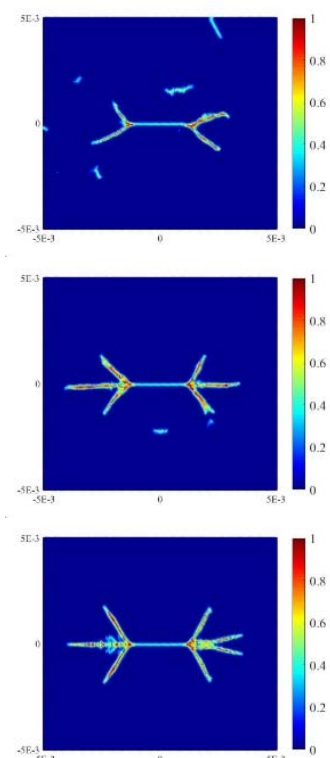

$1.5 \mu \mathrm{s}$
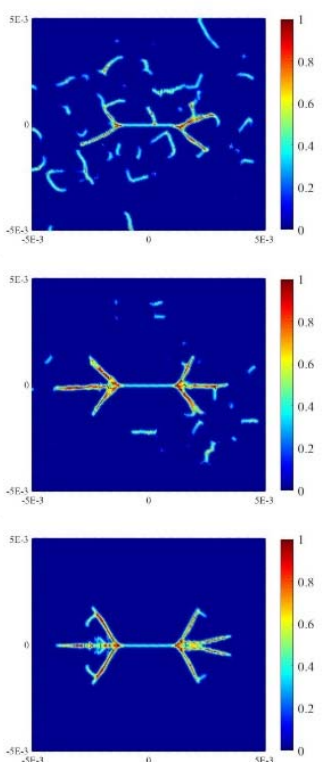

$2 \mu s$

Fig. 26. Crack propagation for the horizontal pre-existing crack case for different percentages of silicon carbide with 100 grains. From the top to the bottom the percentages of the silicon carbide are: $50 \%, 10 \%$, and $0 \%$.
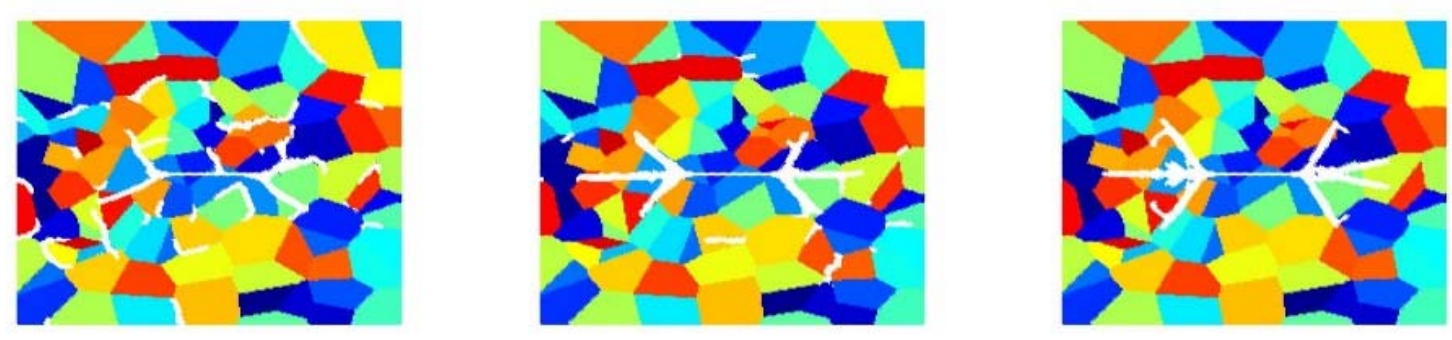

(a) Crack propagation showing the distribution of crystals.
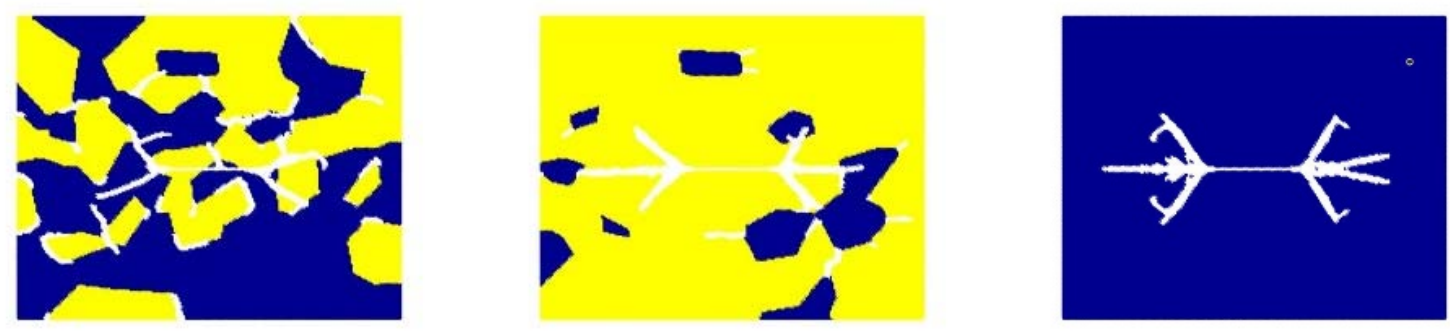

(b) Crack propagation showing the material composition (blue represents silicon carbide).

Fig. 27. Crack propagation for the horizontal pre-existing crack case with crystals and material composition at $2 \mu$ s for $50 \%, 10 \%$, and $0 \%$ silicon carbide with 100 grains. 

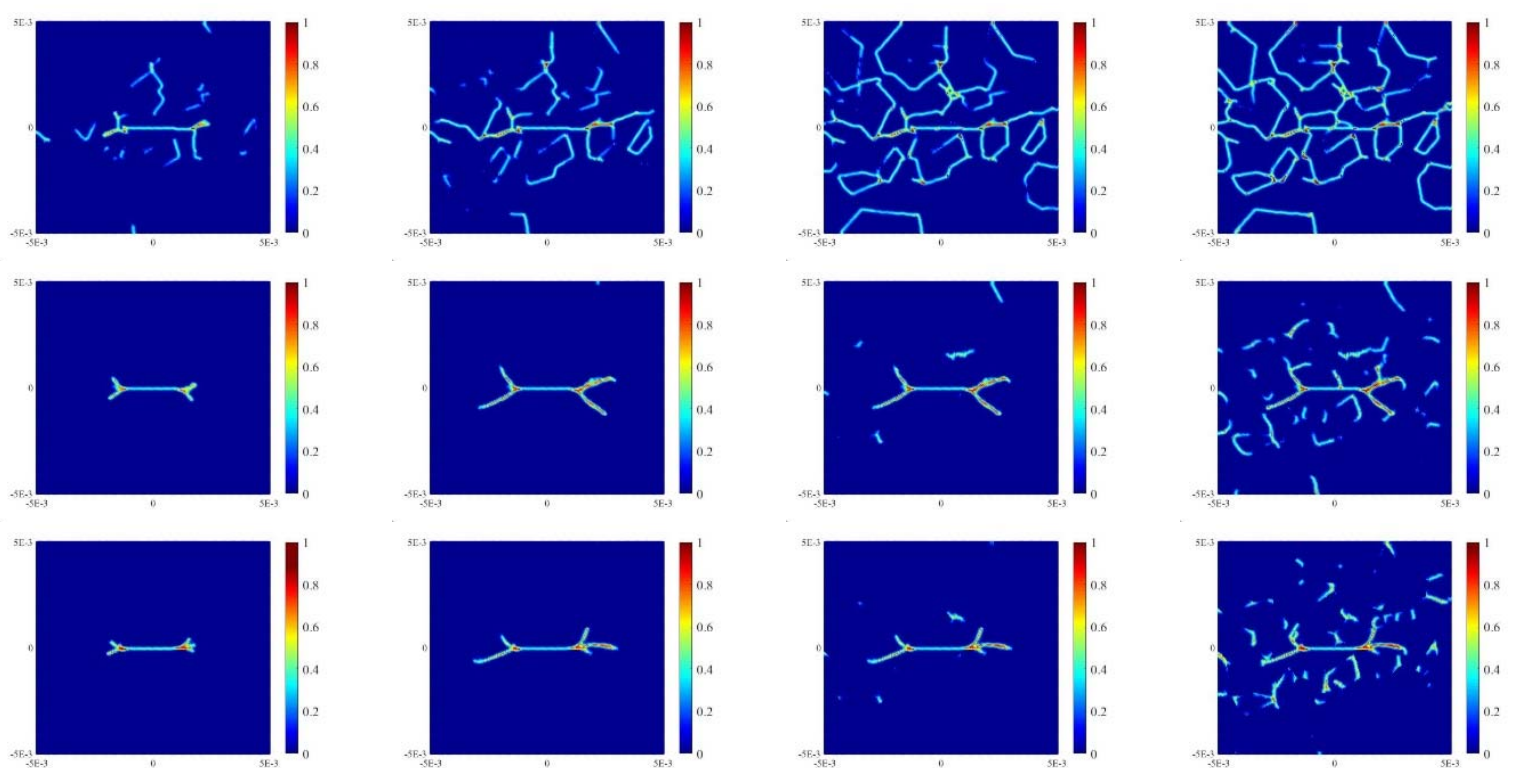

$0.5 \mu \mathrm{s}$

$1 \mu s$

$1.5 \mu \mathrm{s}$

$2 \mu \mathrm{s}$

Fig. 28. Crack propagation for the horizontal pre-existing crack case for different values of GBC with 100 grains and 50\% silicon carbide. From the top to the bottom the values of GBC are: $0.5,1$, and 2 .
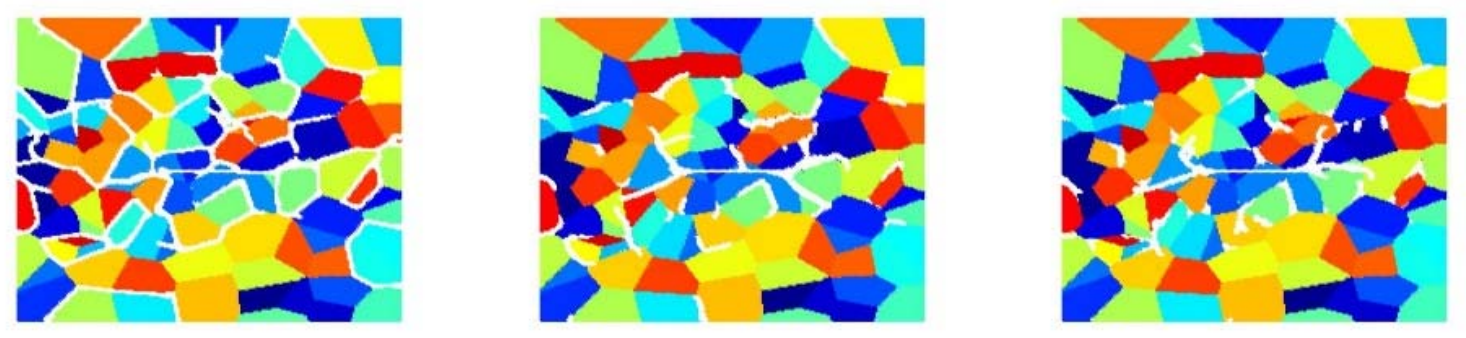

(a) Crack propagation showing the distribution of crystals.
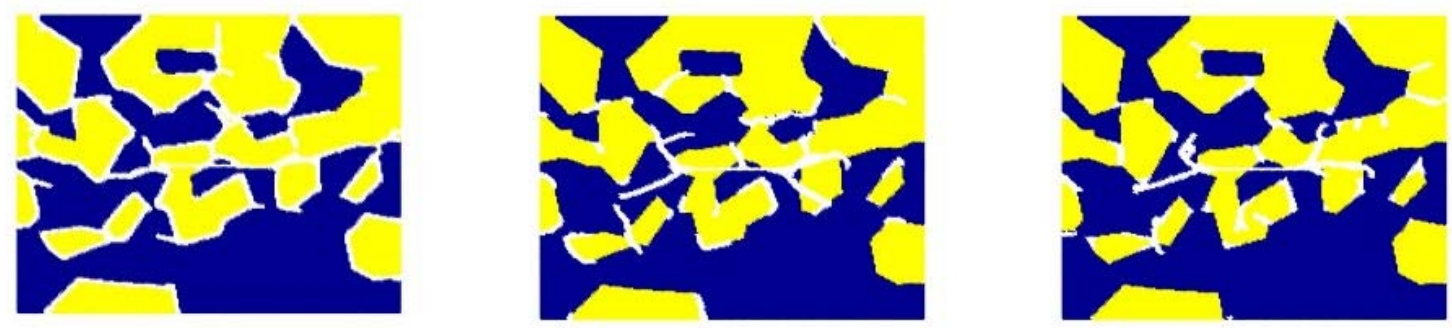

(b) Crack propagation showing the material composition (blue represents silicon carbide).

Fig. 29. Crack propagation for the horizontal pre-existing crack case with crystals and material composition at $2 \mu \mathrm{s}$ for $0.5,1$, and 2 GBC with 100 grains.

\section{Conclusion}

In this study, thermally-induced crack propagation in polycrystalline materials was simulated using peridynamics. The accuracy of the model was tested and verified with three static cases under thermal loading for three different material models including isotropic, single cubic crystal, and cubic polycrystal. The PD analysis displacement results were identical to 
conventional FEM results. Afterwards, a cubic polycrystalline material with two different types of pre-existing cracks, i.e. vertical and horizontal, were analyzed by applying dynamic cooling temperature change. The effects of grain size and grain boundary strength were investigated. The effect of grain size was much more obvious in the horizontal crack case in which branching crack behaviour was observed. For the vertical crack case, initial main cracks propagated in a self-similar manner. Grain boundary strength has a significant influence on crack behaviour. As expected, for weaker grain boundaried intergranular fracture pattern was observed whereas for stronger grain boundaries transgranular crack pattern was more dominant. Finally, a ceramic material made of two different materials, i.e. silicon carbode and alumina, was considered. As opposed to cubic polycrystalline case, crack branching occurred for both vertical and horizontal cracks. Crack branching was much more significant when the silicon carbide ratio is higher due to the difference in coefficients of thermal expansion of two different materials. As in the cubic polycrystalline case, as the grain boundary strength decreases, the fracture mode turns into an intergranular character. The reached conclusions are in good agreement with the reference studies available in the literature.

\section{Acknowledgement}

The authors gratefully acknowledges the financial support from China Scholarship Council (No. 201806680018) and University of Strathclyde.

\section{References}

[1] De Meo, D., Zhu, N. and Oterkus, E., 2016. Peridynamic modeling of granular fracture in polycrystalline materials. Journal of Engineering Materials and Technology, 138(4), p.041008.

[2] Case, E.D., Smyth, J.R. and Hunter, O., 1980. Grain-size dependence of microcrack initiation in brittle materials. Journal of Materials Science, 15(1), pp.149-153.

[3] Kuszyk, J.A. and Bradt, R.C., 1973. Influence of grain size on effects of thermal expansion anisotropy in MgTi2O5. Journal of the American Ceramic Society, 56(8), pp.420423.

[4] Adams, J.W., Nakamura, H.H., Ingel, R.P. and Rice, R.W., 1985. Thermal Expansion Behaviro of single-Crystal Zirconia. Journal of the American Ceramic Society, 68(9), pp.C228.

[5] Groeber, M.A., Haley, B.K., Uchic, M.D., Dimiduk, D.M. and Ghosh, S., 2006. 3D reconstruction and characterization of polycrystalline microstructures using a FIB-SEM system. Materials Characterization, 57(4-5), pp.259-273.

[6] Ludwig, W., Reischig, P., King, A., Herbig, M., Lauridsen, E.M., Johnson, G., Marrow, T.J. and Buffiere, J.Y., 2009. Three-dimensional grain mapping by x-ray diffraction contrast tomography and the use of Friedel pairs in diffraction data analysis. Review of Scientific Instruments, 80(3), p.033905.

[7] Herbig, M., King, A., Reischig, P., Proudhon, H., Lauridsen, E.M., Marrow, J., Buffière, J.Y. and Ludwig, W., 2011. 3-D growth of a short fatigue crack within a polycrystalline 
microstructure studied using combined diffraction and phase-contrast X-ray tomography. Acta Materialia, 59(2), pp.590-601.

[8] Geraci, G. and Aliabadi, M.H., 2017. Micromechanical boundary element modelling of transgranular and intergranular cohesive cracking in polycrystalline materials. Engineering Fracture Mechanics, 176, pp.351-374.

[9] Camacho, G.T. and Ortiz, M., 1996. Computational modelling of impact damage in brittle materials. International Journal of solids and structures, 33(20-22), pp.2899-2938.

[10] Ortiz, M. and Pandolfi, A., 1999. Finite-deformation irreversible cohesive elements for three-dimensional crack-propagation analysis. International journal for numerical methods in engineering, 44(9), pp.1267-1282.

[11] Tvergaard, V. and Hutchinson, J.W., 1992. The relation between crack growth resistance and fracture process parameters in elastic-plastic solids. Journal of the Mechanics and Physics of Solids, 40(6), pp.1377-1397.

[12] Lin, L., Wang, X. and Zeng, X., 2017. The role of cohesive zone properties on intergranular to transgranular fracture transition in polycrystalline solids. International Journal of Damage Mechanics, 26(3), pp.379-394.

[13] Sfantos, G.K. and Aliabadi, M.H., 2007. A boundary cohesive grain element formulation for modelling intergranular microfracture in polycrystalline brittle materials. International journal for numerical methods in engineering, 69(8), pp.1590-1626.

[14] Benedetti, I. and Aliabadi, M.H., 2013. A three-dimensional cohesive-frictional grainboundary micromechanical model for intergranular degradation and failure in polycrystalline materials. Computer Methods in Applied Mechanics and Engineering, 265, pp.36-62.

[15] Sfantos, G.K. and Aliabadi, M.H., 2007. Multi-scale boundary element modelling of material degradation and fracture. Computer Methods in Applied Mechanics and Engineering, 196(7), pp.1310-1329.

[16] Geraci, G. and Aliabadi, M.H., 2018. Micromechanical modelling of cohesive thermoelastic cracking in orthotropic polycrystalline materials. Computer Methods in Applied Mechanics and Engineering, 339, pp.567-590.

[17] Geraci, G. and Aliabadi, M.H., 2019. Micromechanical modeling of cohesive thermoelastic steady-state and transient cracking in polycrystalline materials. International Journal for Numerical Methods in Engineering, 117(12), pp.1205-1233.

[18] Zeng, X. and Li, S., 2010. A multiscale cohesive zone model and simulations of fractures. Computer methods in applied mechanics and engineering, 199(9-12), pp.547-556.

[19] Qian, J. and Li, S., 2011. Application of multiscale cohesive zone model to simulate fracture in polycrystalline solids. Journal of Engineering Materials and Technology, 133(1), p.011010.

[20] Li, S., Zeng, X., Ren, B., Qian, J., Zhang, J. and Jha, A.K., 2012. An atomistic-based interphase zone model for crystalline solids. Computer Methods in Applied Mechanics and Engineering, 229, pp.87-109. 
[21] Galvis, A.F. and Sollero, P., 2016. 2D analysis of intergranular dynamic crack propagation in polycrystalline materials a multiscale cohesive zone model and dual reciprocity boundary elements. Computers \& Structures, 164, pp.1-14.

[22] Sukumar, N., Srolovitz, D.J., Baker, T.J. and Prévost, J.H., 2003. Brittle fracture in polycrystalline microstructures with the extended finite element method. International Journal for Numerical Methods in Engineering, 56(14), pp.2015-2037.

[23] Kamaya, M., Kawamura, Y. and Kitamura, T., 2007. Three-dimensional local stress analysis on grain boundaries in polycrystalline material. International Journal of Solids and Structures, 44(10), pp.3267-3277.

[24] Silling, S.A., 2000. Reformulation of elasticity theory for discontinuities and long-range forces. Journal of the Mechanics and Physics of Solids, 48(1), pp.175-209.

[25] Madenci, E. and Oterkus, S., 2016. Ordinary state-based peridynamics for plastic deformation according to von Mises yield criteria with isotropic hardening. Journal of the Mechanics and Physics of Solids, 86, pp.192-219.

[26] Amani, J., Oterkus, E., Areias, P., Zi, G., Nguyen-Thoi, T. and Rabczuk, T., 2016. A non-ordinary state-based peridynamics formulation for thermoplastic fracture. International Journal of Impact Engineering, 87, pp.83-94.

[27] Gao, Y. and Oterkus, S., 2019. Ordinary state-based peridynamic modelling for fully coupled thermoelastic problems. Continuum Mechanics and Thermodynamics, 31(4), pp.907937.

[28] Oterkus, E. and Madenci, E., 2012. Peridynamics for failure prediction in composites. In 53rd AIAA/ASME/ASCE/AHS/ASC Structures, Structural Dynamics and Materials Conference 20th AIAA/ASME/AHS Adaptive Structures Conference 14th AIAA (p. 1692).

[29] Oterkus, E., Barut, A. and Madenci, E., 2010. Damage growth prediction from loaded composite fastener holes by using peridynamic theory. In 51st AIAA/ASME/ASCE/AHS/ASC Structures, Structural Dynamics, and Materials Conference 18th AIAA/ASME/AHS Adaptive Structures Conference 12th (p. 3026).

[30] Oterkus, S. and Madenci, E., 2014. Fully coupled thermomechanical analysis of fiber reinforced composites using peridynamics. In 55th AIAA/ASMe/ASCE/AHS/SC Structures, Structural Dynamics, and Materials Conference-SciTech Forum and Exposition 2014.

[31] Javili, A., Morasata, R., Oterkus, E. and Oterkus, S., 2019. Peridynamics review. Mathematics and Mechanics of Solids, 24(11), pp.3714-3739.

[32] Ghajari, M., Iannucci, L. and Curtis, P., 2014. A peridynamic material model for the analysis of dynamic crack propagation in orthotropic media. Computer Methods in Applied Mechanics and Engineering, 276, pp.431-452.

[33] Askari, E., Bobaru, F., Lehoucq, R.B., Parks, M.L., Silling, S.A. and Weckner, O., 2008. Peridynamics for multiscale materials modeling. In Journal of Physics: Conference Series (Vol. 125, No. 1, p. 012078). IOP Publishing. 
[34] De Meo, D., Diyaroglu, C., Zhu, N., Oterkus, E. and Siddiq, M.A., 2016. Modelling of stress-corrosion cracking by using peridynamics. International Journal of Hydrogen Energy, 41(15), pp.6593-6609.

[35] Zhu, N., De Meo, D. and Oterkus, E., 2016. Modelling of granular fracture in polycrystalline materials using ordinary state-based peridynamics. Materials, 9(12), p.977.

[36] Madenci, E. and Oterkus, E., 2014. Peridynamic Theory. In Peridynamic Theory and Its Applications (pp. 19-43). Springer, New York, NY.

[37] Bobaru, F., Yang, M., Alves, L.F., Silling, S.A., Askari, E. and Xu, J., 2009.

Convergence, adaptive refinement, and scaling in 1D peridynamics. International Journal for Numerical Methods in Engineering, 77(6), pp.852-877.

[38] Dirras, G.F., Djemia, P., Roussigne, Y. and Jackson, K.M., 2004. Investigating the elastic properties of $\beta$-SiC films. Materials Science and Engineering: A, 387, pp.302-306.

[39] Pharr, M., Katoh, Y. and Bei, H., 2006. Dependence of Fracture Toughness on Crystallographic Orientation in Single-Crystalline Cubic $(\beta)$ Silicon Carbide. Journal of Undergraduate Research, 6. 


\section{Appendix}

The convergence study is performed for the dynamic analysis of ceramic made of two materials case. During the m-convergence test, $100 \times 100,200 \times 200$, and $400 \times 400 \mathrm{PD}$ points are chosen and the number of the PD points is the only variable. The GBC is fixed at 1 and two different types of pre-existing cracks are considered. The results of the three cases are computed and the plots are shown in Fig. A1 to Fig. A4.
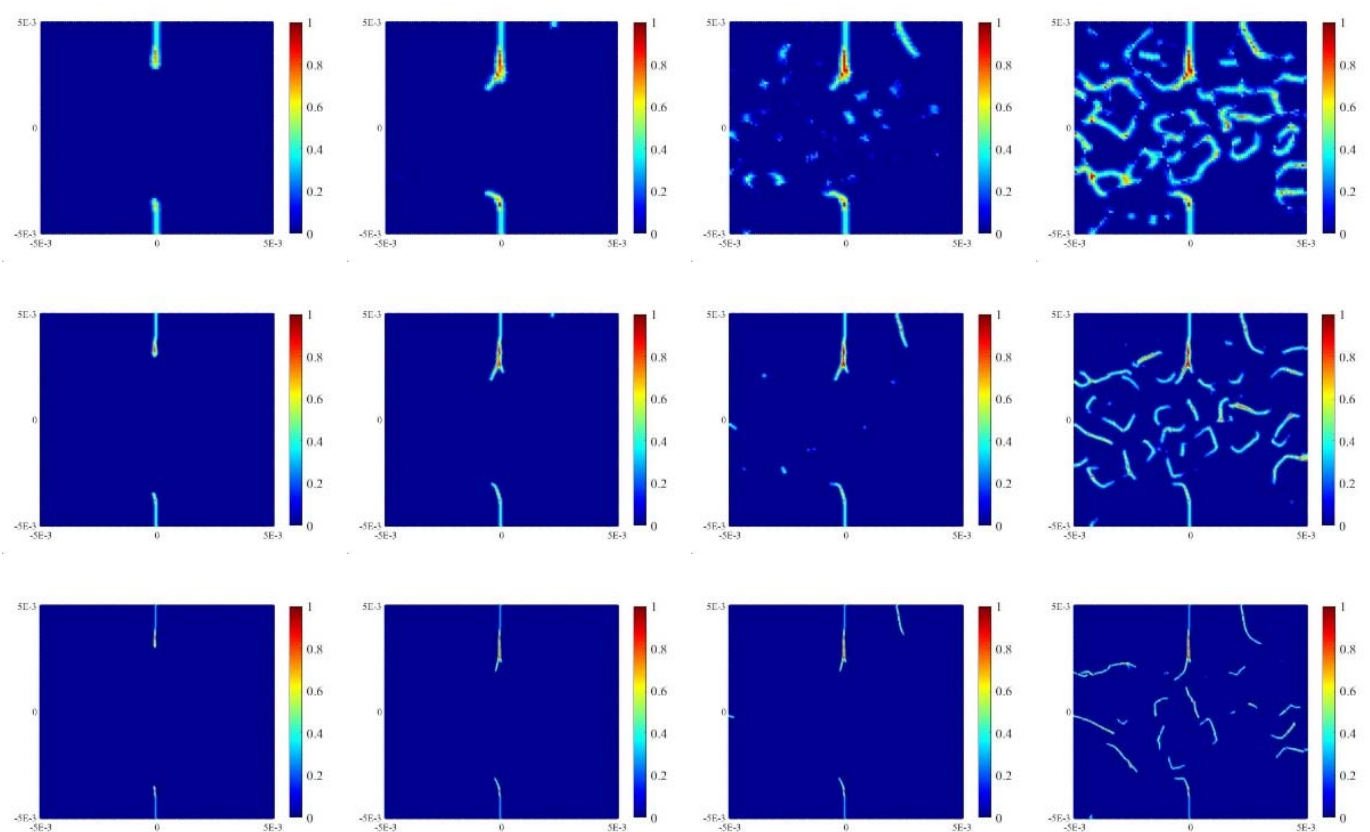

$0.5 \mu s$

$1 \mu \mathrm{s}$

$1.5 \mu \mathrm{s}$

$2 \mu s$

Fig. A1. Crack propagation for the vertical pre-existing crack case for different numbers of PD points. From the top to the bottom the number of the points is: $100 \times 100,200 \times 200$, and $400 \times 400$. 

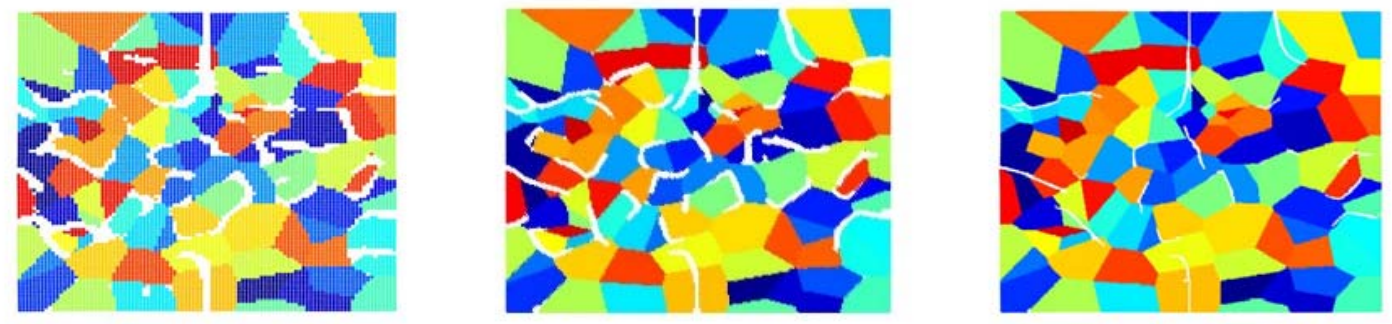

(a) Crack propagation showing the distribution of crystals
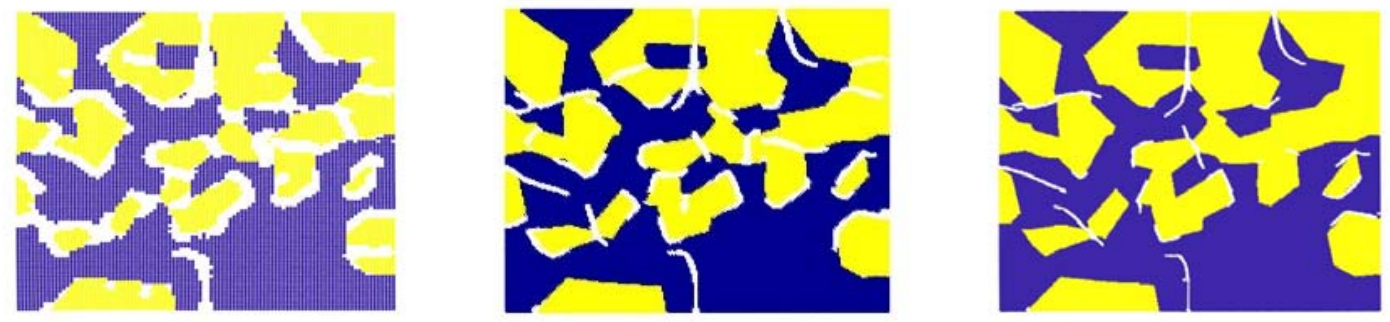

(b) Crack propagation showing the material composition (blue represents silicon carbide)

Fig. A2. Crack propagation for the vertical pre-existing crack case at $2 \mu$ s for $100 \times 100$, $200 \times 200$, and $400 \times 400$ points.
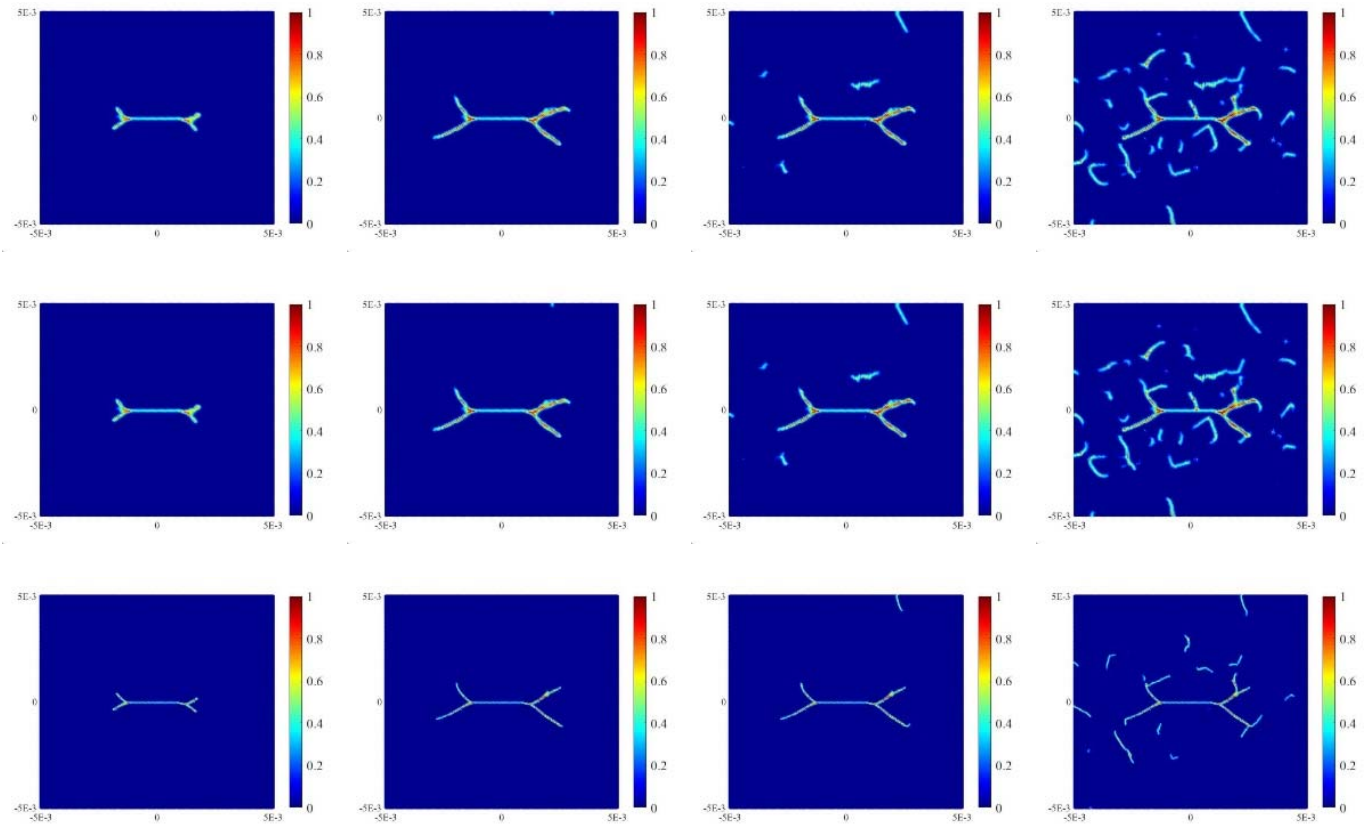

$0.5 \mu s$

$1 \mu \mathrm{s}$

$1.5 \mu \mathrm{s}$

$2 \mu s$

Fig. A3. Crack propagation for the horizontal pre-existing crack case for different numbers of PD points. From the top to the bottom the number of the points is: $100 \times 100,200 \times 200$, and $400 \times 400$. 

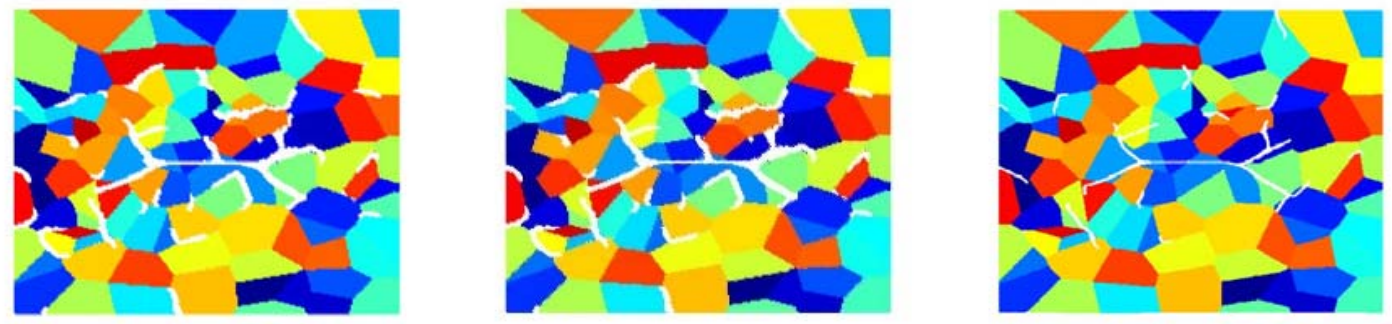

(a) Crack propagation showing the distribution of crystals
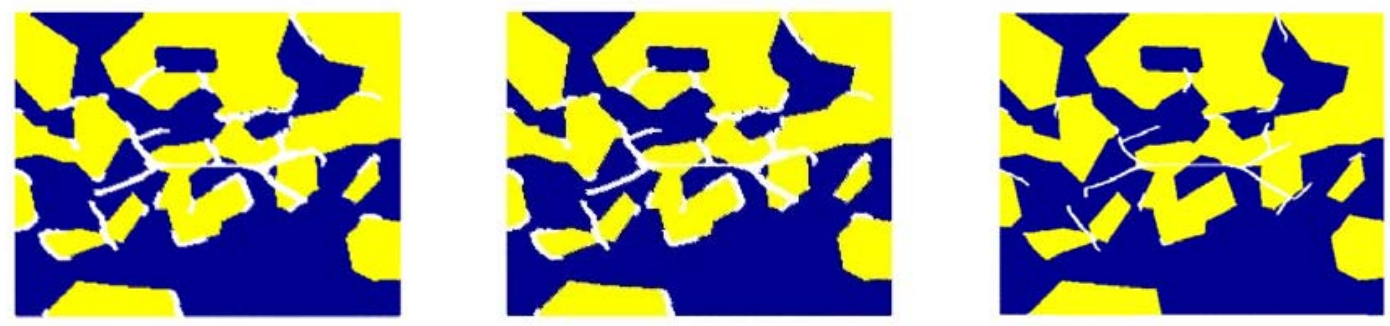

(b) Crack propagation showing the material composition (blue represents silicon carbide)

Fig. A4. Crack propagation for the horizontal pre-existing crack case at $2 \mu$ s for $100 \times 100$, $200 \times 200$, and $400 \times 400$ points.

From the plots, it can be seen that with the increase of the number of material points, the damage patterns especially the major fractures are not strongly influenced. It can be concluded that the discretization will affect the resolutions of the plots rather than the main features of the crack propagation. Compared to $100 \times 100$ (low resolution) and $400 \times 400$ (time-consuming), $200 \times 200$ PD points is a reasonable choice to keep the critical features of the crack as well as high resolutions of the plots.

For the $\delta$-convergence, as shown in Fig. A5-A8, the horizon size does not have significant influence on the pattern of the main crack. Therefore, $\delta=3 d x$ is selected since this is suggested horizon size according to [1] and [36] which can provide accurate results with less computational time. 

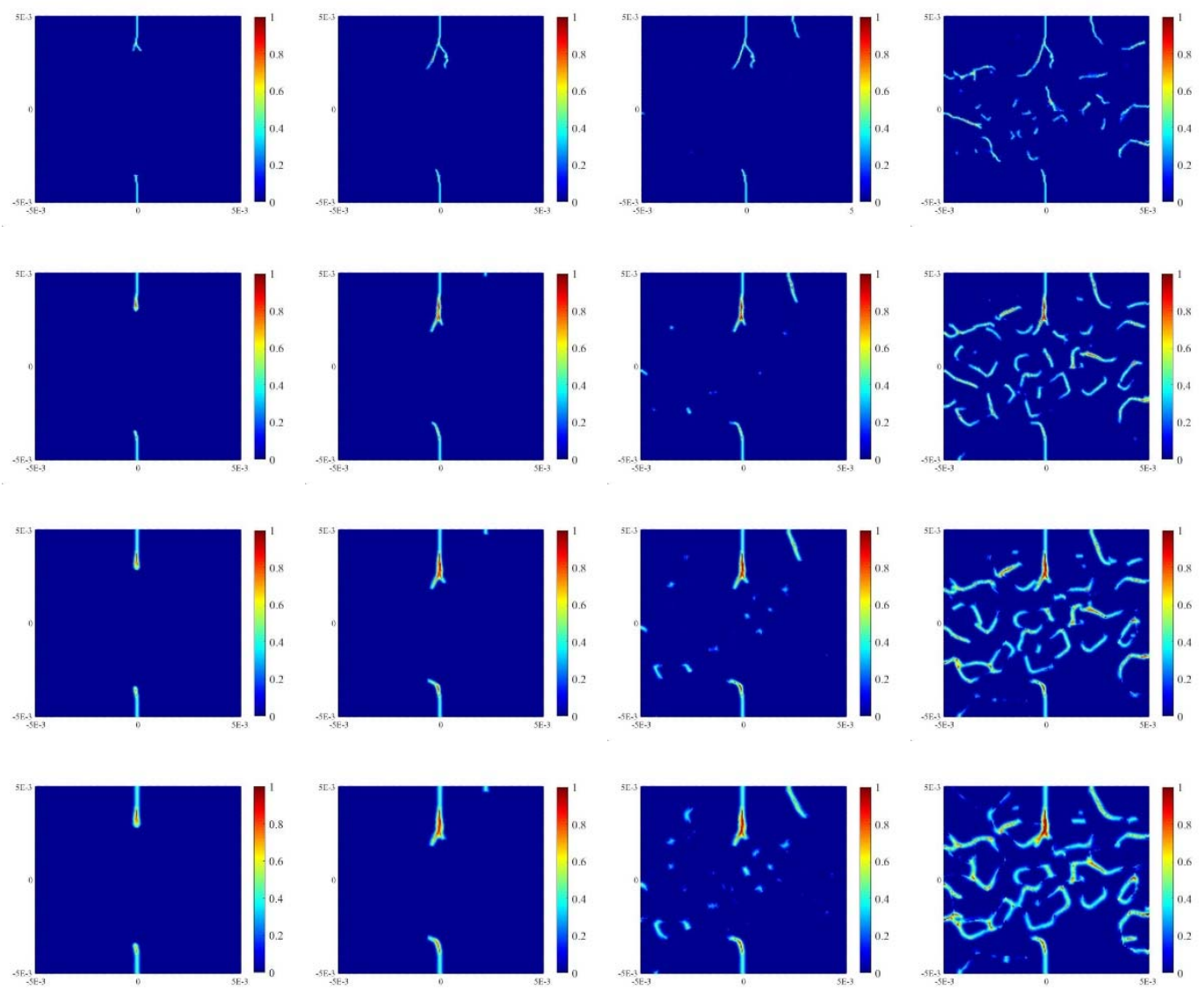

$0.5 \mu s$

$1 \mu s$

$1.5 \mu s$

$2 \mu s$

Fig. A5. Crack propagation for the vertical pre-existing crack case for different horizon sizes. From the top to the bottom the horizon size is: $2 d x, 3 d x, 4 d x$, and $5 d x$.
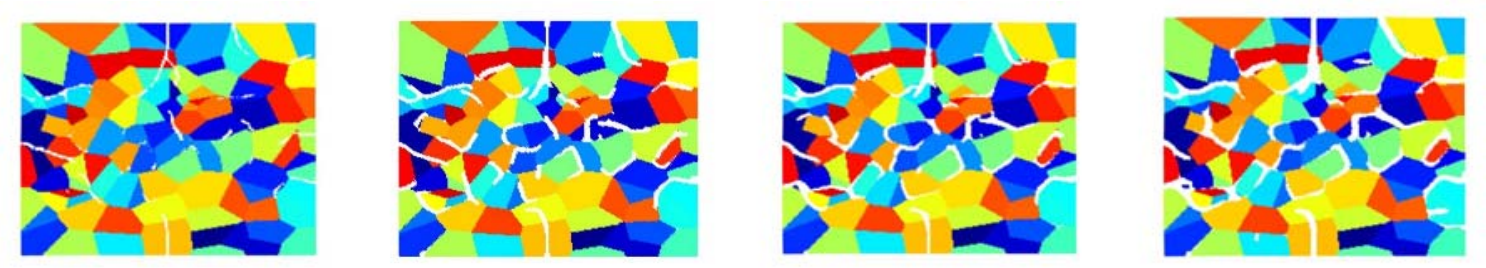

(a) Crack propagation showing the distribution of crystals
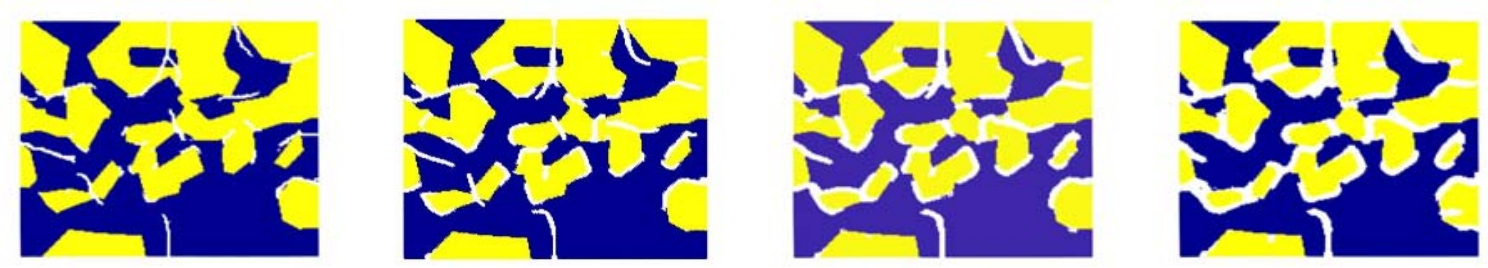

(b) Crack propagation showing the material composition (blue represents silicon carbide)

Fig. A6. Crack propagation for the vertical pre-existing crack case at $2 \mu$ s for $2 d x, 3 d x$, $4 d x$, and $5 d x$ horizon sizes. 

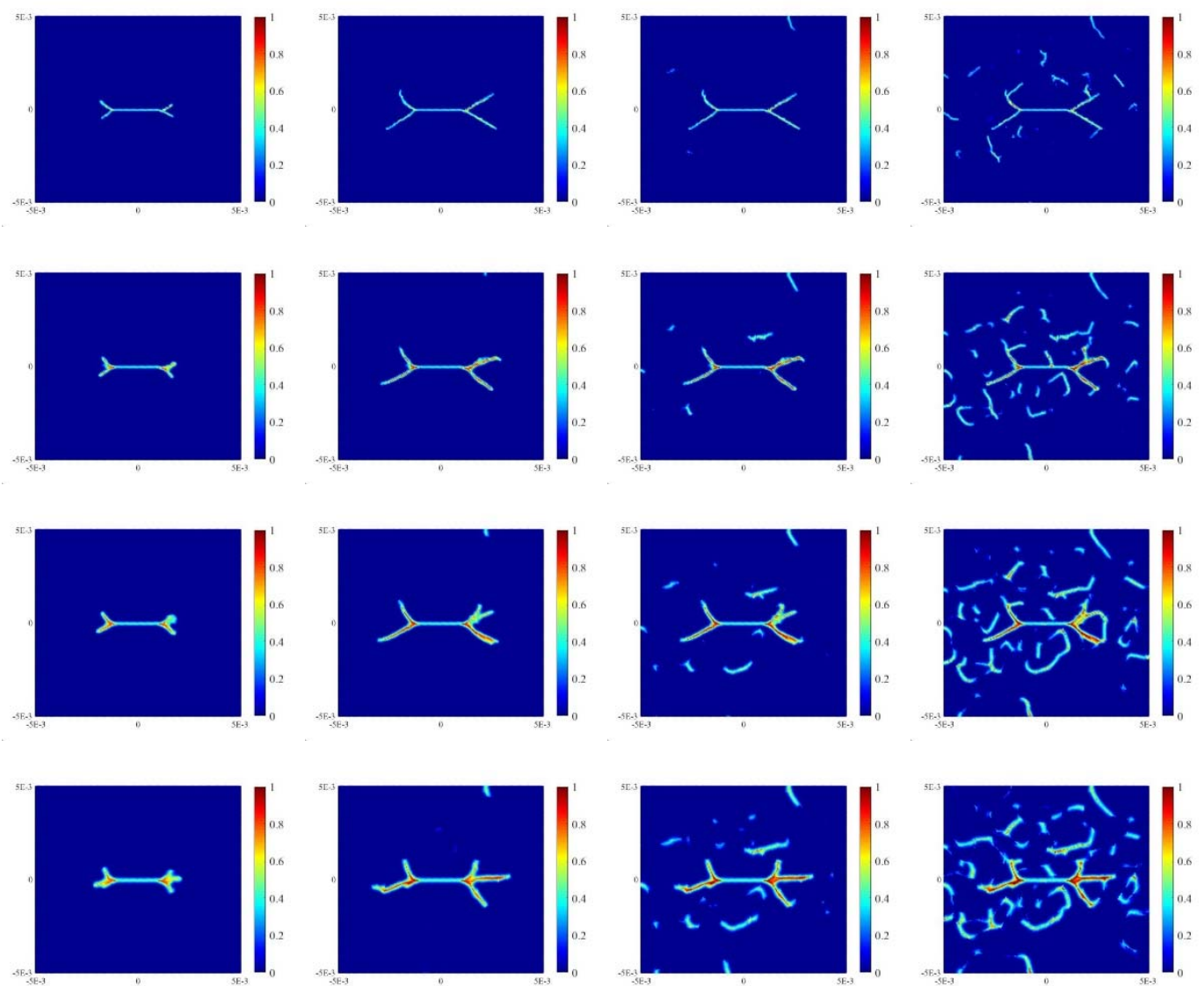

$0.5 \mu s$

$1 \mu s$

$1.5 \mu s$

$2 \mu s$

Fig. A7. Crack propagation for the horizontal pre-existing crack case for different horizon sizes. From the top to the bottom the horizon size is: $2 d x, 3 d x, 4 d x$, and $5 d x$.
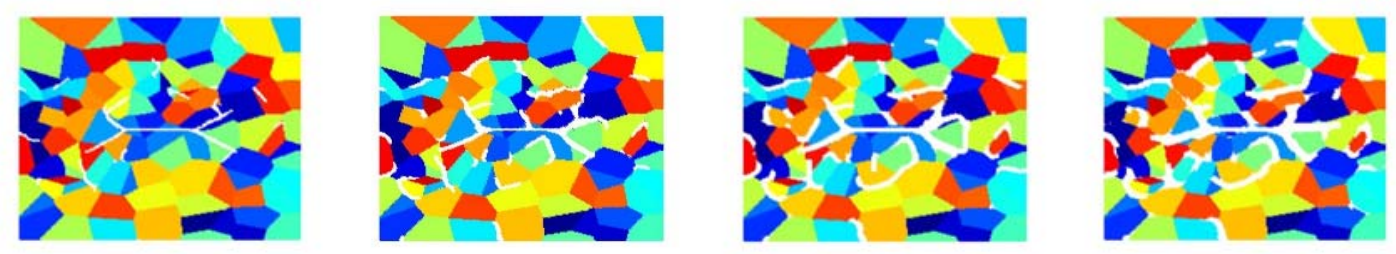

(a) Crack propagation showing the distribution of crystals
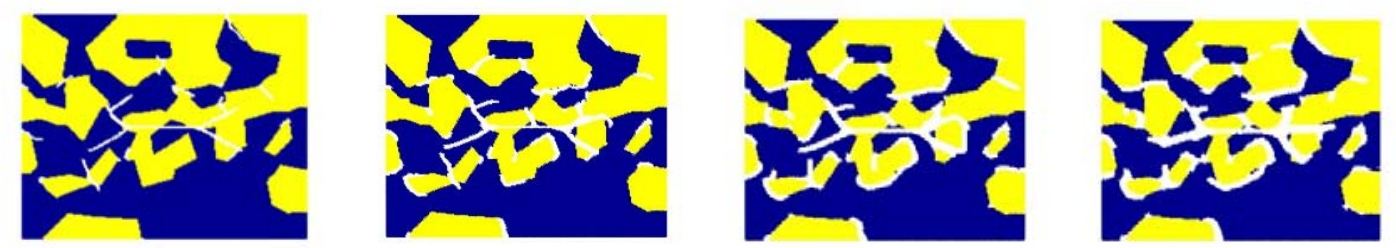

(b) Crack propagation showing the material composition (blue represents silicon carbide)

Fig. A8. Crack propagation for the horizontal pre-existing crack case at $2 \mu$ s for $2 d x, 3 d x$, $4 d x$, and $5 d x$ horizon sizes. 